\title{
Paediatric functional abdominal pain disorders
}

Nikhil Thapar ${ }^{1,2,14 凶}$, Marc A. Benninga ${ }^{3}$, Michael D. Crowell ${ }^{4}$, Carlo Di Lorenzo ${ }^{5}$, Isabelle Mack, Samuel Nurko ${ }^{7}$, Miguel Saps ${ }^{8}$, Robert J. Shulman ${ }^{9}$, Hania Szajewska ${ }^{10}$, Miranda A. L. van Tilburg ${ }^{11,12,13}$ and Paul Enck ${ }^{6 凶}$

Abstract | Paediatric functional abdominal pain disorders, currently referred to as disorders of gut-brain interaction, comprise irritable bowel syndrome, functional dyspepsia, abdominal migraine and functional abdominal pain not otherwise specified, as defined by the Rome IV diagnostic criteria. Functional abdominal pain disorders are common disorders with a prevalence of $3-16 \%$ depending on country, age and sex. A greater understanding of aetiopathogenesis and pathophysiology is emerging and includes intestinal components (inflammation, motility and the microbiota), central factors (psychological aspects, sensitization and/or differences in connectivity or activity of certain brain regions) as well as extrinsic factors (infections). In particular, the timing of disruption of the microbiota-gut-brain axis seems to be important. Diagnosis is challenging but is primarily based on clinical symptoms and exclusion of other organic causes, with an emphasis on avoiding unnecessary invasive diagnostic procedures. The available pharmacological interventions are limited in children and, therefore, management has focused on combined approaches, including mind-targeted interventions (hypnotherapy and cognitive behavioural therapy), diet (probiotics) and percutaneous electrical nerve field stimulation. The evidence for their clinical efficacy, although limited, is favourable, with positive impacts on symptoms and overall quality of life. The coming decades hold promise for improved understanding and management of these enigmatic disorders.

Hyperalgesia An abnormally heightened sensitivity to pain. 凶e-mail:nikhil.thapar@ health.qld.gov.au; paul.enck@uni-tuebingen.de https://doi.org/10.1038 s41572-020-00222-5
Dedication: We dedicate this manuscript to the memory of Paul E. Hyman, MD, who passed away on 7 August 2020. Paul was a true pioneer and master of the field of functional gastrointestinal disorders and dedicated much of his career to caring for children suffering from such conditions. A great family man, friend, colleague, mentor and inspiration to so many.

Functional abdominal pain disorders (FAPDs) are some of the most commonly encountered disorders in childhood, affecting up to $25 \%$ of all children and infants worldwide ${ }^{1}$. Given their biopsychosocial aetiology involving complex interactions within the gut-brain axis, FAPDs are currently referred to as 'disorders of gutbrain interaction ${ }^{2}$. Furthermore, the gut-brain axis is now accurately referred to as the 'microbiota-gut-brain axis', reflecting an explosion in our understanding of the magnitude, complexity, role and interactions of the microbial populations hosted within the lumen of the gastrointestinal tract ${ }^{3,4}$.

Akin to adults, paediatric FAPDs are subclassified utilizing the Rome IV criteria into a number of clinically distinct entities, namely irritable bowel syndrome
(IBS), functional dyspepsia, abdominal migraine and functional abdominal pain not otherwise specified $(\text { FAP-NOS })^{4}$. FAPDs are frequently characterized by the presence of visceral hyperalgesia as well as increased central perception of visceral stimuli leading to disability, which seems to occur as the final outcome of sensitizing psychosocial factors and medical factors superimposed on a background of genetic predisposition and early-life events (FIG. 1). Early life is likely to include all childhood and adolescent stages where growth as well as the structural and functional development of organs occurs, although the vulnerability of the gut-brain-microbiota axis seems to be highest during the perinatal period and first years of life. FAPDs are grouped according to symptom profile, which differs based on the section of gastrointestinal tract that is primarily involved (for example, functional dyspepsia versus IBS) or depending on similarities with other conditions such as headache migraine (BOX 1).

IBS describes a symptom profile reflecting distal gastrointestinal tract involvement; abdominal pain in IBS can be associated with defaecation or a change in the frequency or form of the stool but does not resolve with resolution of any associated constipation. Paediatric IBS 


\author{
Author addresses \\ ${ }^{1}$ Neurogastroenterology and Motility Unit, Department of Paediatric Gastroenterology, \\ Great Ormond Street Hospital NHS Foundation Trust, London, UK. \\ 'UCL Great Ormond Street Institute of Child Health, London, UK. \\ ${ }^{3}$ Emma Children's Hospital, Amsterdam University Medical Center, Amsterdam, \\ Netherlands. \\ ${ }^{4}$ Department of Gastroenterology and Hepatology, Mayo Clinic, Scottsdale, AZ, USA. \\ ${ }^{5}$ Division of Pediatric Gastroenterology, Nationwide Children's Hospital, Columbus, \\ $\mathrm{OH}, \mathrm{USA}$. \\ ${ }^{6}$ University Hospital Tübingen, Department of Internal Medicine VI: Psychosomatic \\ Medicine and Psychotherapy, Tübingen, Germany. \\ 'Boston Children's Hospital, Harvard medical School, Boston, MA, USA. \\ ${ }^{8}$ Division of Pediatric Gastroenterology, Hepatology and Nutrition, University of Miami, \\ Miller School of Medicine, Miami, FL, USA. \\ ${ }^{9}$ Children's Nutrition Research Center, Baylor College of Medicine, Texas Children's \\ Hospital, Houston, TX, USA. \\ ${ }^{10}$ Department of Pediatrics, The Medical University of Warsaw, Warsaw, Poland. \\ ${ }^{11}$ College of Pharmacy and Health Sciences, Campbell University, Buies Creek, NC, USA. \\ ${ }^{12}$ Division of Gastroenterology and Hepatology, University of North Carolina, Chapel Hill, \\ NC, USA. \\ ${ }^{13}$ School of Social Work, University of Washington, Seattle, WA, USA. \\ ${ }^{14}$ Present address: Queensland Children's Hospital, Paediatric Gastroenterology, \\ Hepatology and Liver Transplant, Brisbane, Australia.
}

Postprandial fullness

Unpleasant sensation thought

to emanate from the stomach

for a considerable period after

eating a meal giving the feeling

of the persistence of food. can be further divided into subtypes analogous to adults based on the predominant stool pattern ${ }^{5}$ (BOX 2).

Functional dyspepsia describes a symptom profile predominantly from the proximal gastrointestinal tract involving epigastric abdominal pain and/or bothersome postprandial fullness and/or early satiation. Similar to the criteria in adults, subtypes of paediatric functional dyspepsia now recognized include postprandial distress syndrome and epigastric pain syndrome, accepting a frequent overlap between them ${ }^{6-8}$.
Abdominal migraine is characterized by the presence of paroxysmal prolonged episodes of intense, acute periumbilical, midline or diffuse abdominal pain. These painful episodes are typically incapacitating and interfere with normal activities and occur in a stereotypical pattern (that is, in a set form or pattern, for example, with regards to the presence or form of aura and the escalation of symptoms, severity and frequency) for the individual patient. The episodes are often but not always associated with other symptoms (such as anorexia, nausea, vomiting, headache, photophobia and pallor), which might precede or coincide with the duration of pain, and such symptomatic episodes may be separated by weeks to months.

FAP-NOS accounts for those instances of episodic pain or continuous abdominal pain that does not occur solely during physiological events and does not meet criteria for the other FAPDs ${ }^{4}$.

For paediatric cases, the Rome IV criteria heralded a number of key milestones with respect to defining and characterizing these disorders. First, the term 'functional abdominal pain disorders' superseded the previously used terminology 'abdominal pain-related functional gastrointestinal disorders', allowing better focus for clinicians on the four subclassifications. Second, in the Rome IV classification, the previous criterion phrase "no evidence of an inflammatory, anatomic, metabolic, or neoplastic process that explains the subject's symptoms" has been replaced with "after appropriate medical evaluation, the symptoms cannot be attributed to another medical condition"9. This modification has allowed greater autonomy to the clinicians to use their expertise to decide whether selective testing, or, in some cases,

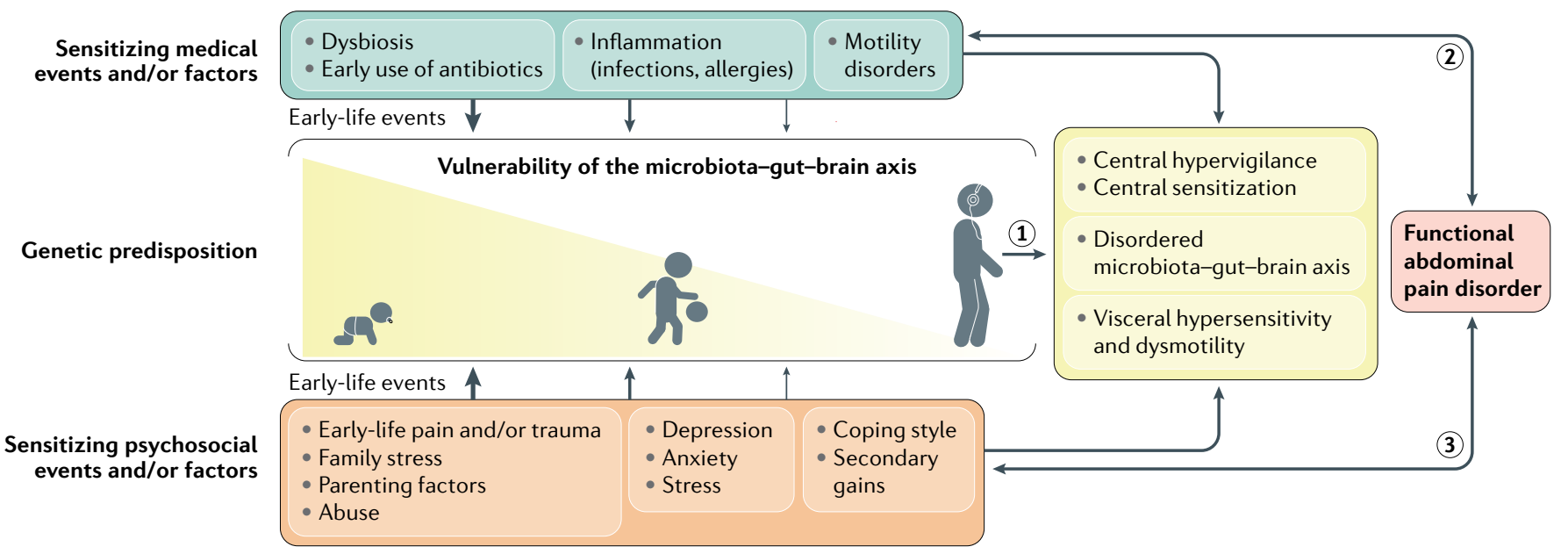

Fig. 1 | Summary of the aetiopathogenesis and pathophysiology of FAPDs. This figure illustrates the likely contribution of genetic predisposition as well as of sensitizing psychosocial and medical events to the disruption of the structure and/or function of the microbiota-gut-brain axis and to the development of the core disturbances of functional abdominal pain disorders (FAPDs), namely visceral hypersensitivity (with or without motor disturbances) and central hypervigilance. Although insults can occur at any time in life, early postnatal life represents an especially vulnerable period to disrupt the gut-brain axis, yet the core disturbances may not be evident until later in life (1). The reasons for this delay are not clear and may relate to other, as yet unknown and, presumably, additive or threshold-lowering factors such as changes in the hypothalamic-pituitary- adrenal axis, sex hormones, or changes in the brain structure or function. These changes might explain why children in the second decade of life are most vulnerable to the development of FAPDs. Bidirectional arrow (2) denotes the possibility of FAPDs and their consequences (both medical and psychosocial, for example, deconditioning, stress and anxiety) driving medical events or factors (such as mast cell degranulation and interaction with nerves) through mechanisms such as stress. Bidirectional arrow (3) denotes the possibility of FAPDs and their consequences driving psychosocial events/factors (for example, anxiety, stress, depression, poor coping and familial stress), which may further exacerbate the condition. Details of 'early-life events' associated with the development of FAPDs are explained and discussed in the manuscript. 


\section{Box 1 | Rome IV diagnostic criteria for functional abdominal pain disorders}

\section{Irritable bowel syndrome}

The criteria must be fulfilled for at least 2 months and include all of the following.

- Abdominal pain at least 4 days per month associated with defaecation and/or a change in the frequency of stool and/or a change in the appearance of stool

- Abdominal pain does not resolve with resolution of the constipation (children in whom the pain resolves have functional constipation, not irritable bowel syndrome)

- After appropriate evaluation, the symptoms cannot be fully explained by another medical condition

\section{Functional dyspepsia}

The criteria must be fulfilled for at least 2 months before diagnosis and must include one or more of the following bothersome symptoms at least 4 days per month.

- Postprandial fullness

- Early satiation

- Epigastric pain or burning not associated with defaecation

- After appropriate evaluation, the symptoms cannot be fully explained by another medical condition

\section{Abdominal migraine}

The criteria must be fulfilled for at least 6 months before diagnosis and include all of the following occurring at least twice.

- Paroxysmal episodes of intense, acute periumbilical, midline or diffuse abdominal pain lasting 1 hour or more (should be the most severe and distressing symptom)

- Episodes are separated by weeks to months; the pain is incapacitating and interferes with normal activities; stereotypical pattern and symptoms in the individual patient

- The pain is associated with two or more of the following: anorexia, nausea, vomiting, headache, photophobia or pallor

- After appropriate evaluation, the symptoms cannot be fully explained by another medical condition

\section{Functional abdominal pain not otherwise specified}

The criterial must be fulfilled for at least 2 months before diagnosis and at least four times per month and include all of the following.

- Episodic or continuous abdominal pain that does not occur solely during physiological events (for example, eating and menses)

- Insufficient criteria for irritable bowel syndrome, functional dyspepsia or abdominal migraine

- After appropriate evaluation, the abdominal pain cannot be fully explained by another medical condition

Adapted with permission from REF. ${ }^{4}$, Elsevier. sensory function or motor function of nerves) underlies these conditions. The term is not, nor should be, used to imply a 'non-organic' condition.

This Primer summarizes the current progress in our understanding of paediatric FAPDs in terms of prevalence and aetiopathogenesis, with a specific focus on early-life influences and programming. Furthermore, we discuss the current diagnostic approach and highlight the therapeutic options for the management of these disorders, with a particular focus on IBS and FAP-NOS. Finally, we also discuss the directions for future research that will help understand the pathophysiology and better treat these disorders.

\section{Epidemiology}

Although FAPDs are undoubtedly prevalent in children, epidemiological studies have been hampered owing to the lack of appropriate diagnostic criteria. The development of diagnostic criteria and the subclassification of the original vague entity 'recurrent abdominal pain in children' have only become available as of 2005 with the advent of the Rome II criteria ${ }^{14}$. Since then, the two further iterations of the Rome criteria have had substantial changes compounding the problem with elucidating and comparing prevalence data from different studies ${ }^{4,8,9}$. One limiting factor that prevents comparability of the data across the globe is the use of different tools for assessment, which has been overcome only in the past 15 years. Nearly $80 \%$ of the studies applying the Rome III criteria used the Questionnaire on Paediatric Gastrointestinal Symptoms-Rome III criteria for assessment, whereas studies applying the Rome I criteria or Rome II criteria used a large variety of instruments.

Additionally, the reliability and validity of methods used to identify the different FAPDs are limited. For example, studies comparing diagnostic reliability have demonstrated marked differences between an independent physician's diagnosis and a child's or a parent's reporting of the diagnostic symptoms ${ }^{15-17}$. The latter reporting is especially critical as parents might have limited knowledge of their children's bowel habits, especially in adolescence ${ }^{18,19}$.

These challenges in epidemiological studies are evident in the literature; for example, a large populationbased birth cohort (born from 1994 through 1996) in Sweden found that $26 \%$ of all children suffered from recurrent abdominal pain on at least one out of three assessment points, namely early childhood (pooling data from questionnaires at 1-2 years of age) and at 12 and 16 years of age ${ }^{20}$. Approximately $20 \%$ of children in the 16-year age group reported recurrent abdominal pain but only $12 \%$ could be categorized into one of the FAPDs (IBS, functional dyspepsia or functional abdominal pain) according to the Rome III criteria ${ }^{20}$. Furthermore, a cross-sectional retrospective chart review of 106 paediatric patients who completed standardized medical histories as part of their evaluation for chronic abdominal pain showed that significantly more patients were likely to be diagnosed with functional dyspepsia $(84.9 \%$ versus $52.8 \%$ ) and IBS (69.8\% versus $34 \%$ ) by the Rome IV criteria than by the Rome III criteria ${ }^{8}$. Conversely, another study found a lower prevalence of any FAPDs 
( $8.2 \%$ versus $10.4 \%)$ and IBS (2.3\% versus $5.1 \%)$ but a higher prevalence of functional dyspepsia (3.0\% versus $1.0 \%$ ) diagnosed by the Rome IV criteria than by the Rome III criteria ${ }^{21}$. Similar significant differences in the accurate classification of cases were found between the Rome II and Rome III criteria ${ }^{22}$.

The literature regarding epidemiological data on functional dyspepsia and abdominal migraine is relatively sparse. In the Swedish birth cohort study discussed above, of the $12 \%$ diagnosed with an abdominal pain-related functional gastrointestinal disorder based on the Rome III criteria, $6 \%$ were diagnosed with IBS and $3 \%$ each were diagnosed with functional abdominal pain and functional dyspepsia ${ }^{20}$. The presence of abdominal migraine was not assessed in these children and information about functional dyspepsia at earlier time points (that is, at 1-2 years) and at 12 years of age was not available. A cross-sectional observational study in Colombia that employed the Rome IV criteria revealed a prevalence of $3 \%$ and $0.5 \%$ for functional dyspepsia and abdominal migraine, respectively ${ }^{21}$. A 2015 metanalysis of 58 studies from across the world (excluding Africa and Australia) reported a pooled prevalence of $13.5 \%$ (95\% CI 11.8-15.3) for all FAPDs and a pooled prevalence of $4.5 \%$ for functional dyspepsia and $1.5 \%$ for abdominal migraine $^{23}$. Notably, a major variation was reported in the prevalence of FAPDs across studies, ranging from $1.6 \%$ to $41.2 \%$. However, the pooled prevalence from each geographical region ranged only between $10.5 \%$ (Europe) and $16.8 \%$ (South America). Interestingly, in a community-based study in the USA on 949 children using parental reporting based on the Questionnaire on Paediatric Gastrointestinal Symptoms-Rome III criteria, abdominal migraine had the highest prevalence amongst FAPDs, with a reported prevalence of $9.2 \%$ compared with $2.8 \%$ for IBS and $0.2 \%$ for functional dyspepsia ${ }^{24}$.

Population studies in children and adolescents (3-16 years of age) worldwide reported a pooled global

\section{Box 2 | Subclassification of functional abdominal pain disorders}

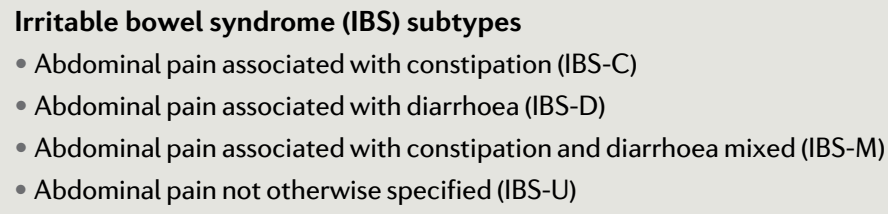

\section{Functional dyspepsia subtypes}

- Postprandial distress syndrome includes bothersome postprandial fullness or early satiation that prevents finishing a regular meal; supportive features include upper abdominal bloating, postprandial nausea or excessive belching

- Epigastric pain syndrome includes all of the following: bothersome pain that is severe enough to interfere with normal activities or burning localized to the epigastrium; the pain is not generalized or localized to other abdominal or chest regions and is not relieved by defaecation or passage of flatus; supportive criteria can include burning pain without a retrosternal component or pain commonly induced or relieved by the ingestion of a meal but can occur while fasting

\section{Other functional abdominal pain disorders}

- Abdominal migraine and functional abdominal pain not otherwise specified are not subclassified

Adapted with permission from REF. ${ }^{4}$, Elsevier. prevalence of 13.8 for IBS, which varied between $0 \%$ and $25.7 \%$ based on the ROME I-III criteria (FIG. 2). The study reported a pooled prevalence of $2.3 \%$ for FAP-NOS, which ranged between $0.3 \%$ and $5.2 \%$. Most of the studies were conducted in North America, South America and Asia, followed by Europe. The pooled prevalence of IBS was found to be highest in Asia (16.2\%) and was reported to be $4.8 \%$ in the USA and $4.2 \%$ in Europe. Asia also had the highest prevalence of FAP-NOS (3.1\%), whereas this was reported to be $1.6 \%$ in the USA and $1.7 \%$ in Europe. Only one study was conducted in Africa, suggesting a prevalence of $5.6 \%{ }^{25}$, not allowing a firm statement to be made.

\section{Risk factors}

Sex. Studies from across the world have reported a predominance of FAPDs in girls, both in adolescents and in younger children ${ }^{26}$. A metanalysis ${ }^{23}$ was performed on epidemiological studies reporting the prevalence or incidence of FAPD according to the different ROME criteria as well as other previously used criteria or defined by the presence of non-organic abdominal pain in children with at least three episodes of abdominal pain and/or weekly episodes of abdominal pain and/or a symptom duration of at least 3 months. All but 2 of the 24 studies reported a predominance of FAPD in girls. The pooled prevalence data also revealed a significantly higher proportion of FAPDs among girls than among boys (15.9\% versus $11.5 \%$, pooled OR 1.5 , 95\% CI 1.3-1.7; $P<0.01)$. Notably, this difference was also evident at a pre-pubertal age (10 years) (9.9\% girls versus $7.7 \%$ boys, OR 1.4, 95\% CI 1.16-1.79; $P<0.001)^{26}$.

In addition, some studies also reported a higher prevalence of IBS and FAP-NOS in girls, consistent with the findings of the meta-analysis ${ }^{23}$, whereas a few other studies reported no difference ${ }^{5,27,28}$. The pooled prevalence of IBS was $17.2 \%$ in girls and $15.0 \%$ in boys and the pooled prevalence of FAP-NOS was 3\% in girls and $2 \%$ in boys. Currently, data are lacking on the role of sex in the prevalence of functional dyspepsia and abdominal migraine. Across both conditions, findings have been inconsistent, with a number of studies suggesting no difference in prevalence between sexes ${ }^{24,29}$ and a number of studies reporting otherwise $\mathrm{e}^{22,30}$.

Age. The role of age in the aetiology of FAPD is inconclusive, mainly owing to the heterogeneous results of studies reporting the effects of age. For IBS, nine studies reported no differences in prevalence with age, six studies reported an increase in prevalence with age and four studies reported a decrease in prevalence with age. These findings are in line with those of a meta-analysis conducted in 2015, which pooled data from 36 studies worldwide involving children aged $<12$ years and $>12$ years, showing no significant difference in the prevalence of FAPDs across different age groups ${ }^{23}$. Additionally, one study reported a peak prevalence of IBS (12\%) at 11 years of age and a decreased prevalence in children both younger and older than 11 years of age $^{31}$. The heterogeneity of results from studies investigating age might be due to the diverse age groups included in these studies. 


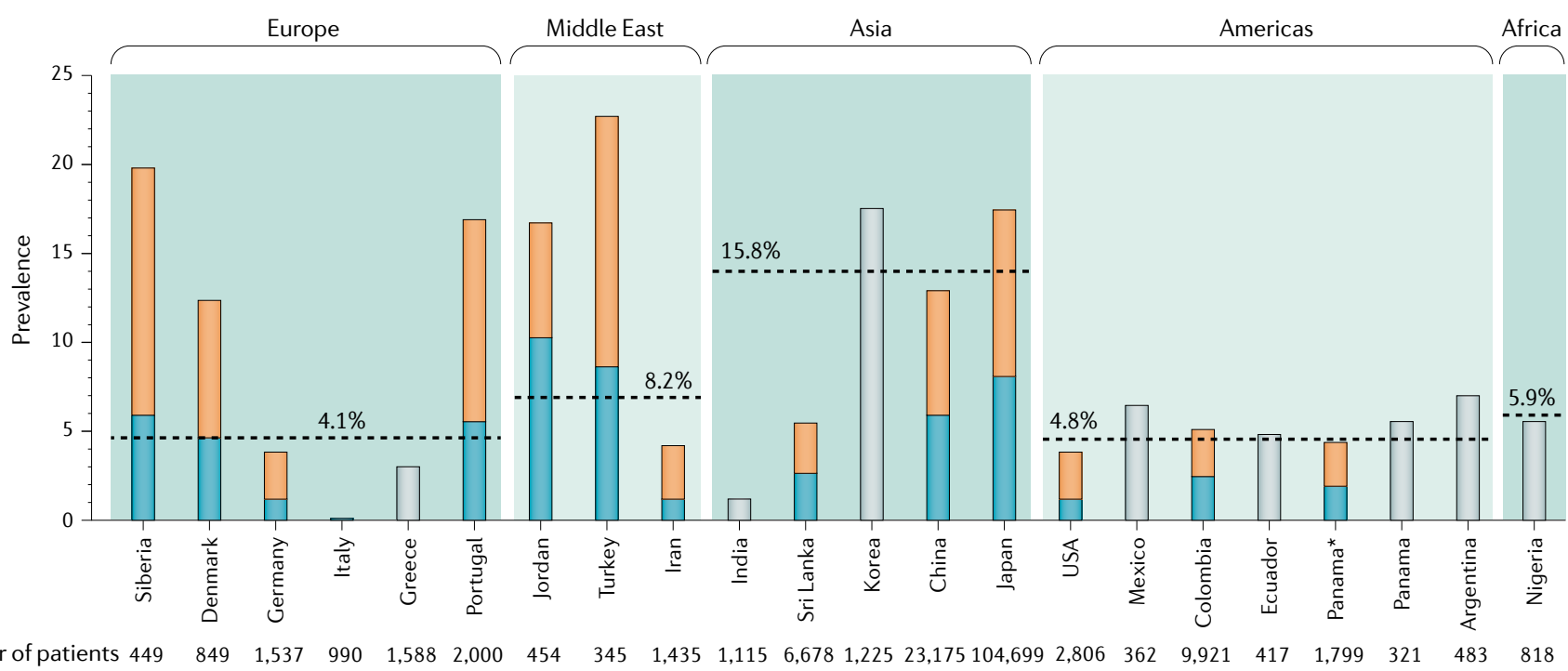

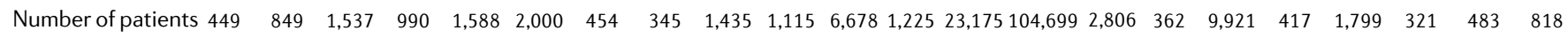

$$
\square \text { Percentage of girls } \square \text { Percentage of boys } \quad \square \text { Percentage of boys and girls } \quad \text {-.. Pooled prevalence }
$$

Fig. 2 IBS prevalence in children. Prevalence of irritable bowel syndrome (IBS) in children and adolescents in different jurisdictions of the world, according to single studies as well as meta-analyses ${ }^{23}$. The regions are sorted according to continents, with pooled prevalence for continents represented. The total number of individuals in the cohort are listed. In few areas, prevalence data in girls versus boys were available, whereas in India, Korea and Mexico, only data on the overall prevalence was available (denoted by grey bars). Notably, not all epidemiological studies differentiated IBS and other functional abdominal pain disorders as studies were conducted over a long period of time (between 1958 and 2013), and they used different diagnostic criteria for paediatric functional abdominal pain disorders, introducing substantial variation. Additionally, assessment tools (questionnaires versus interviews) and assessment levels (birth cohorts, population samples and school samples) may contribute to this variability in prevalence. *Panama and other countries: Ecuador, El Salvador, Nicaragua and Mexico.

Psychosocial factors. Although family history, other somatic symptoms (for example, headaches, dizziness and muscle pains), mental health status and socioeconomic variables are often acknowledged as associated factors for all types of FAPD, their effects on prevalence are mostly unreported. Several studies reported that children and adolescents with FAPDs have a poor mental health status, that is, higher levels of anxiety and/or depression and/or emotional problems, higher stress levels or experienced stressful events, and have lower quality of life (see below) than healthy children ${ }^{32}$. Up to $50 \%$ of children with FAPD are reported to have clinically relevant anxiety or depression ${ }^{33}$. In addition, girls with FAPDs are more likely to have experienced gastrointestinal infections or surgeries earlier in life. Furthermore, children with FAPDs also exhibit more extraintestinal somatic symptoms, such as headache, fatigue and sleep problems, than healthy children ${ }^{32}$. Social contextual factors such as parental chronic pain have also been linked to increased episodes of pain in children. Indeed, children of mothers with IBS report bothersome gastrointestinal symptoms, miss more days at school and seek health-care professionals more frequently than children in control families ${ }^{34}$. Interestingly, chronic pain in both parents was associated with significantly poorer health in children than having one or neither parent with chronic pain $^{35}$. Extensive literature suggests that using the social learning theory ${ }^{34,36,37}$ and the social communication model of pain ${ }^{38}$ parents can influence their children's perception of pain by modelling their pain behaviour and reinforcing their children's pain complaints with solicitous responses ${ }^{39}$ - children express their pain in the same way as their parents behave with pain.

Genetic factors. A family history of IBS and other FAPDs has been reported as an epidemiological risk factor ${ }^{40-42}$. For IBS, a substantial overlap in the incidence of abdominal symptoms between mothers and their children has been described ${ }^{24}$. This overlap might be related to certain genes but might more likely be due to a number of social factors, including attentive parental response to child pain behaviours ${ }^{36}$. Although twin studies show that the concordance rate of IBS is significantly higher in monozygotic twins (17.2\%) than in dizygotic twins (8.4\%), indicating genetic contribution, the low overall concordance strongly implies that social factors and environmental factors are at play ${ }^{43}$.

Although several studies in adults have indicated a number of susceptibility genes for the development of FAPDs, the results are inconsistent. No similar studies have been reported in children, although a small pilot study suggested that $\sim 16 \%$ of children with IBS have pedigrees suggestive of maternal inheritance, possibly associated with mitochondrial DNA-related dysfunction ${ }^{44}$.

\section{Mechanisms/pathophysiology}

FAPDs are complex disorders that seem to result from disruption of the functional and/or, more subtly, the structural integrity of one or more elements of the 
microbiota-gut-brain axis. The intricate and multifaceted nature of the interactions that underlie these conditions are brought together into a biopsychosocial model (FIG. 1), which is applicable to all FAPDs. Crucial components of this model are the concepts of visceral hypersensitivity and central hypervigilance, although the relative importance of each of these components is unclear and likely to vary between individuals. The role of genetics versus environmental factors is not conclusive and, although both elements seem to be key, neither factor seems sufficient on its own to contribute to the development of FAPDs ${ }^{4}$. Indeed, the pathogenetic model is far from simple and continues to evolve; this section aims to highlight certain aspects of the pathogenesis of FAPDs from our contemporary understanding.

\section{Visceral hypersensitivity}

Visceral hypersensitivity describes a perceptual response (that is, hyperalgesia) to peripheral signals and can be a result of changes in visceral afferent signal processing (reflecting increased visceral afferent input from the gut to the brain) or a consequence of alterations in descending modulation of pain (for example, central sensitization - see below $)^{45}$. Visceral hypersensitivity in children is often manifested by a decreased sensory threshold for pain compared with control children ${ }^{46}$. This gut-specific hypersensitivity might be observed during distension of the bowel (for example, rectal distension in IBS) or in response to exposure to altered chemical composition of the chyme (for example, lipids or acid in functional dyspepsia and bile in IBS), which may, in part, explain responses to treatments such as dietary modification and acid suppression.

Studies involving adults and those using animal models have proposed a number of potential triggers, such as inflammation, infection and stress, for the development of visceral hypersensitivity (FIG. 1). In essence, these triggers can cause changes in mucosal permeability and lead to inflammation, releasing algogenic factors, including serotonin (5-HT), histamine, NGF, proteases and prostaglandins, which are capable of activating receptors on afferent nociceptors (pain-sensing nerves), causing acute pain as well as longer-lasting functional changes (that is, peripheral sensitization) and structural changes (via increased nerve sprouting) key to chronic pain maintenance (FIG. 3). Children seem to be especially susceptible to developing such hypersensitivity early in life.

\section{Central sensitization}

Central sensitization refers to the phenomenon of amplification of pain sensitivity via the enhancement of neuronal function and neural signalling within the central nervous system that elicits pain hypersensitivity and is a well-described mechanism in the context of chronic pain development and maintenance ${ }^{47}$ (FIG. 3). In addition to pain hypersensitivity, central sensitization results in secondary changes in brain activity, which can be detected by electrophysiological or imaging techniques. Individuals with FAPDs may likely have a higher than normal propensity to developing central sensitization of unclear origin. Furthermore, predisposing insults, such as stress, might occur in certain individuals at a time of vulnerability of the relevant central nervous system pathways (for example, critical periods of neuronal growth or development such as in early life or adolescence), making them susceptible to developing FAPDs. A systematic review performed in 2018 involving 12 case-control studies found evidence for secondary hyperalgesia and altered cortical nociceptive processing in children with functional abdominal pain ${ }^{48}$. In 2015, one study demonstrated heightened temporal summation (that is, a pattern of increased perceived pain intensity in response to repetitive stimulation administered at a constant intensity) in adolescents and young adults (8-17 years of age) with FAPDs since childhood and a history of trauma ${ }^{49}$. Using functional MRI to determine rectal distention-induced brain activation, one study showed that adolescent patients with IBS demonstrated more activation of neural networks (such as the salience network) as well as activation of more neural structures (such as the executive network and the emotional arousal network) involved in pain perception and processing than healthy volunteers ${ }^{50}$.

\section{Early-life events}

In paediatric FAPDs, the timing of the insult is more critical than the multiple factors that lead to the disruption of the components of the microbiota-gut-brain axis or than the disruption of the axis itself. In the first two decades of life, particularly from the perinatal period through childhood to adolescence, the key processes of development and maturation of all elements of the gut-brain axis that underpin their functionality are crucial. These key processes include the maturation of neuronal subtypes, the organization and integration of interconnected ganglia of the enteric nervous system to form functional circuits, the development of salience networks within the central nervous system, and the establishment of a functionally mature immune system and microbial populations within the gastrointestinal tract. Interference in these developmental processes at a time of vulnerability (for example, damage or disruption of neural fibres or circuits by surgical transection or inflammation) seems to predispose individuals to FAPDs, presumably by resetting the long-term functional integrity of the neural circuits or the gut-brain axis. Understanding this 'programming' process holds tremendous promise for the prevention and management of FAPDs. An apparent delay ( $>10$ years in some cases) between the insult and manifestation of symptomatic FAPDs has been observed in some cases, predominantly in early adolescence, although the reasons for this predominance are not completely understood ${ }^{42}$. This concept of programming of disease has long been established, for example, coronary heart disease in adult life has been associated with malnutrition in early life (reviewed in REF. ${ }^{51}$ ).

The potency of 'early-life programming' (FIG. 1) is evident in the broad and increasing range of factors (reviewed in $\mathrm{REF}^{42}$ ) that may precede FAPD development. These factors include surgery (for example, for umbilical herniae $^{52}$ and pyloric stenosis ${ }^{53}$ ), bacterial gastrointestinal infections ${ }^{54-56}$, inflammatory or immune-mediated conditions (such as coeliac disease ${ }^{57}$, inflammatory bowel disease ${ }^{11}$ and Henoch-Schönlein purpura ${ }^{58}$ ), and 
Gastric emptying

Measure of how effectively stomach contents are moved into the intestine, displayed as $t_{1 / 2}$ (time at which $50 \%$ of contents have emptied) or as the percentage of contents remaining within the stomach at pre-defined time intervals

(1, 2 and 4 hours)

Gastric arrhythmia or dysrhythmia

Abnormal myoelectrical rhythms of the stomach, a normal rhythm is $\sim 3$ cycles per minute.

Gastric accommodation Vagus nerve-mediated reflex associated with a reduction in gastric tone as well as with an increase in gastric volume and gastric compliance, which allows temporary storage of ingested food before controlled release into the intestine.

Tachygastria

Increased ( $>3$ cycles per minute) rate of electrical pacemaker activity in the stomach

\section{Bradygastria}

Decreased ( $<3$ cycles per minute) rate of electrical pacemaker activity in the stomach

Mixed dysrhythmia Elements of both bradygastria and tachygastria present within an electrogastrography reading. innocuous insults occurring in the first few months of life such as gastric suction at birth ${ }^{59}$, allergy to cow milk $^{60}$ and early use of antibiotics ${ }^{61}$. Experimental models focusing on elements of the microbiota-gut-brain axis, such as the enteric nervous system, suggest that certain subtypes of FAPD are characterized by sustained biological structural disruption or functional disruption that occurred secondary to early-life stress ${ }^{62}$ or physical insults ${ }^{63}$. A similar disruption is observed, for example, in germ-free animals, which fail to establish intestinal microbiota after birth, resulting in the lack of or a malfunctioning enteric nervous system ${ }^{64}$. Abnormalities in enteric nervous system function might also underlie the predisposition of children but not of adults to developing FAPDs after contracting bacterial gastroenteritis in the same epidemic outbreak ${ }^{56}$.

\section{Neuroimmune interactions}

In some children with FAPDs, evidence shows the presence of a low-grade gut inflammation and a role for neuroimmune interactions in the pathogenesis. One study found increased mast cell numbers in close proximity to nerves in the ileocolonic mucosa of children with IBS compared with healthy children. Moreover, the nerve fibre-associated mast cell counts were reported to correlate with the intensity of abdominal pain and frequency of painful episodes ${ }^{65}$. In children with functional dyspepsia, eosinophils and mast cells in the antral region of the stomach were significantly activated in $>50 \%$ of patients and were associated with delayed gastric emptying and gastric arrhythmia or dysrhythmia ${ }^{66}$. Similarly, in a retrospective cohort study, functional dyspepsia in children was strongly associated with duodenal eosinophilia in the absence of endoscopic or routine histological findings ${ }^{67}$. In a group of children with functional dyspepsia, one study found higher numbers of nerve-associated mast cells in a subset of children with atopic disease (that is, children with allergy) than in children without atopic disease. This finding also correlated with increased pain intensity and frequency of abdominal pain episodes ${ }^{65}$, indicating a lower threshold for nociceptor activation. In children with atopic disease, exposure to cow milk resulted in mast cell degranulation and gastric dysrhythmia ${ }^{68}$.

Although immune activation and allergy are implicated in FAPDs, their functions are not proven and are apparent in only a subset of cases. Hence, more evidence is needed to clarify the role of allergy and immune activation in the pathogenesis of FAPDs in children.

\section{Gastrointestinal motility}

Gastrointestinal motility abnormalities have been suggested to contribute to the pathophysiology of FAPDs. Specific underlying motor mechanisms have been defined for subtypes of FAPD, including disorders affecting the upper and lower gastrointestinal tracts. Patients with functional upper gastrointestinal symptoms might have delayed gastric emptying and/or reduced gastric accommodation or neither. Patients with functional lower gastrointestinal symptoms might have delayed, accelerated or normal colonic transit, and/or pelvic floor dyssynergia (disturbance of muscular coordination leading to contraction upon attempts to relax pelvic floor muscles). The fact that a single symptom profile can relate to a range of motility disturbances underlies a failure to establish clear causal relationships between upper or lower gastrointestinal motility abnormalities and symptoms. The functioning of the gastrointestinal tract as a single unit might underlie this lack of causality and, therefore, disorders in a specific segment can produce symptoms in a different area; for example, constipation can lead to delayed gastric emptying. Most studies on gastrointestinal motility have been performed in adults with functional dyspepsia and IBS and the contribution of gastrointestinal motility to abdominal migraine and FAP-NOS remains to be established.

Functional dyspepsia. Children with functional dyspepsia have been shown to have abnormalities in gastric function, with disorders in both gastric emptying and gastric accommodation. These disturbances include delayed gastric emptying, impaired initial distribution of a meal within the stomach, impaired accommodation, antral hypomotility, gastric dysrhythmia (tachygastria, bradygastria and mixed dysrhythmia), and altered duodenojejunal motility ${ }^{69}$. Abnormal gastric emptying or electrical gastric rhythm have been found in up to $70 \%$ of children with functional dyspepsia ${ }^{70}$. Youth (8-17 years of age) with functional dyspepsia based on the Rome IV criteria were found to lack the normal postprandial gastric myoelectrical response or autonomic nervous system response following a liquid meal ${ }^{71}$.

In paediatric patients, nausea might be associated with delayed gastric emptying as measured by scintigraphy $^{72}$. Studies on antral and gastric motility using ultrasonography have described a lower amplitude of antral contractions and antral motility index in children with functional dyspepsia than in healthy children (5.1 versus 8.3) and reported severity scores of abdominal pain to be negatively correlated with the rate of gastric emptying. Abnormalities in gastric myoelectric activity have also been described in children, although their importance in the genesis of symptoms have been difficult to ascertain given the inconsistent correlation between gastric myoelectric abnormalities, gastric emptying and symptoms ${ }^{73}$.

Furthermore, using a nutrient drink test, one study showed that children with dyspepsia had a significantly lower maximum ingested volume (maximum volume tolerated before symptoms such as pain, discomfort, nausea and fullness develop, presumed to result from gastric distention) and significantly slower gastric emptying than controls ${ }^{74}$. Similar results were demonstrated with the water-load test ${ }^{7}$. Studies using a gastric barostat (a gold-standard method to measure gastric accommodation) reported that children with FAP-NOS had a lower pain threshold than healthy children, indicative of the presence of visceral hypersensitivity in these children $^{75}$.

Although the above-mentioned studies suggest abnormalities in different aspects of gastric function, large studies have failed to find a direct correlation between gastric function abnormalities and symptoms in patients with functional dyspepsia ${ }^{76,77}$. 

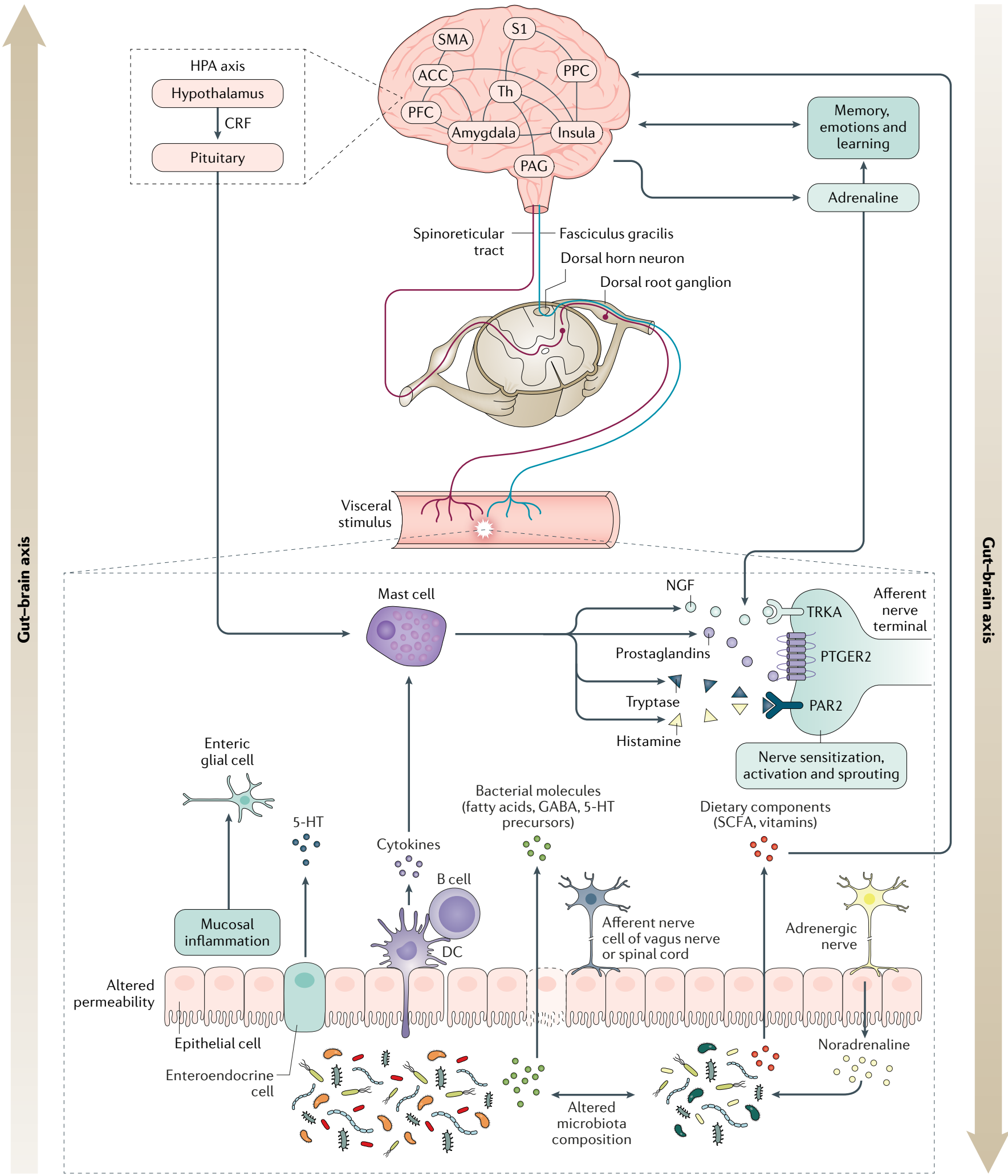

Irritable bowel syndrome. Abnormalities in colonic function and rectal perception have been described in children and adults with $\mathrm{IBS}^{78}$. Colonic function is generally assessed using colonic transit measurements such as radiopaque markers, wireless motility capsule or scintigraphy. Adults with IBS-constipation (IBS-C) have delayed colonic transit and those with IBS-diarrhoea
(IBS-D) have accelerated colonic transit compared with the general population ${ }^{79}$. Unfortunately, most studies investigating colonic function in children have been conducted in a subset of children with severe constipation and therefore do not allow conclusions on colonic transit in IBS-D. In case of abdominal pain, motor alterations (delayed transit) may be associated in some 
4 Fig. 3 | Aetiopathogenesis of hypersensitivity in FAPDs. Central to the biopsychosocial model of functional abdominal pain disorders (FAPDs) is the concept of hypersensitivity occurring at any point from the periphery (gastrointestinal tract) to the central nervous system, often referred to as visceral hypersensitivity and central hypervigilance. The figure illustrates the potential aetiopathogenesis that underlies the disruption of the functional and/or, more subtly, structural integrity of one or more elements of the microbiotagut-brain axis. This axis is a bidirectional communication system enabling gut microorganisms to communicate with the gut (enteric nervous system and immune cells) and the brain as well as the brain with the gut. The mechanisms of interaction are complex and not fully elucidated but include neural (for example, vagus nerve), endocrine, immune and metabolic pathways between the gut and the brain. The gut microbiota might produce neurotransmitters (such as serotonin (5-HT), GABA, noradrenaline, dopamine and acetylcholine), which can cross the mucosal layer of the intestines and influence the enteric nervous system and, indirectly, the brain. Microbial metabolic products (for example, short-chain fatty acids (SCFAs)) may also exert central effects. Signalling from gut to brain is also mediated by immune-mediated production of cytokines, such as IL-1 and IL-6, which can travel via the bloodstream to the brain. In the hypothalamus, these cytokines can activate the hypothalamic-pituitary-adrenal (HPA) axis, bringing about the release of cortisol, a potent activator of the stress system. The HPA axis can also substantially impact the brain-gut-microbiota axis. Brain structures, such as the amygdala, integrate both memory, emotions and learning as well as pain signals (via the periaqueductal grey (PAG)) and stress (prefrontal cortex (PFC) and anterior cingulate cortex (ACC)) and feed them into a matrix of further brain centres such as the insula and the thalamus (Th), which then integrate with signals in the somatosensory cortex (S1, S2 and SMA) from the periphery. DC, dendritic cell; PPC, posterior parietal cortex.

patients with constipation ${ }^{80}$. Three patterns of colonic motility - normal motility, segmental dysmotility or total colonic dysmotility - have been demonstrated in children with constipation ${ }^{81}$. Unfortunately, discriminating symptoms such as pain, constipation and diarrhoea have not always been possible between patients with different colonic motilities. Indeed, pain tends to occur independently of the presence of stool retention and is an important diagnostic marker of IBS. In addition, children with IBS have been shown to have decreased rectal compliance and contractile response to meals ${ }^{82}$.

So far, no clear relationship has been demonstrated between motility abnormalities and symptoms in children with all FAPDs ${ }^{83}$. Whether the observed gastrointestinal motor abnormities are important or a cause or effect of the FAPD is yet to be determined. Indeed, treatment focusing only on improving gastric motility abnormalities (such as improving bowel transit or gastric emptying) does not provide complete symptom relief, indicating the importance of the sensory abnormalities that may accompany motor dysfunction. Nevertheless, owing to the invasive nature of the testing procedures, motor dysfunction is not well investigated both in children and in adults.

\section{Microbiota}

Growing evidence indicates that gut microbiota diversity and composition contribute to FAPDs, particularly IBS $^{84,85}$. However, longitudinal interventional studies establishing the causality between symptoms and the reported cross-sectional dysbiosis are lacking. A 2019 systematic review ${ }^{86}$ assessed gut microbiota composition in healthy individuals and in patients with IBS, both adults and children. Twenty-four studies, most involving adults with various subtypes of IBS (IBS-D, IBS-C and mixed type IBS) and only three studies (reported in two articles) investigating children, met the inclusion criteria. Most studies used faecal sampling owing to its non-invasive nature and for convenience purposes, whereas two studies involving adults assessed mucosal microbiota (via biopsy samples). Overall, the systematic review found a decrease in the abundance of Bifidobacterium spp. and Faecalibacterium spp., and particularly of Faecalibacterium prausnitzii, which is associated with anti-inflammatory effects ${ }^{87}$, in patients with IBS compared with healthy adults. Additionally, the abundance of uncultured protective bacteria, such as Clostridiales I, was decreased, whereas that of potentially harmful bacteria, such as Lactobacillaceae spp., Enterobacteriaceae spp. and Bacteroides spp., were increased in patients with IBS compared with healthy individuals. In addition, the diversity of faecal microbiota was either decreased or remained unchanged in patients with IBS. The authors of the review concluded that their findings were inconsistent and attributed the lack of a standardized approach for microbiota sample identification, collection and processing as a possible reason for this inconsistency. The role of the gut microbiota in symptom induction has been further supported by studies showing that faecal microbiome composition or metabolome composition can predict the patients likely to benefit from the diet ${ }^{88,89}$. Hence, further studies are needed that investigate early-life factors such as mode of delivery (vaginal birth versus caesarean section), diet (breast-feeding versus formula-feeding) and medication use (particularly antibiotics or proton pump inhibitors), all of which are linked to dysbiosis ${ }^{90}$. In addition to bacteria, fungi or viruses might also play a part in FAPD pathophysiology. However, the available data on fungal microbiota dysbiosis or viral microbiota dysbiosis are limited ${ }^{91-93}$, and current studies focus mainly on inflammatory bowel disease ${ }^{94}$ rather than on IBS. Additionally, no studies investigating the role of microbiota in other FAPDs are available to date.

\section{Nutrition}

Diet plays a vital part in children with FAPDs, with up to $93 \%$ identifying at least one food and/or food type as worsening gastrointestinal symptoms, without evidence of food allergy ${ }^{95,96}$. Children with FAPDs frequently self-implement additional dietary strategies such as removing ingredients from the foods they eat ${ }^{95-97}$. Nowadays, the internet is the main source of information, although, unfortunately, the quality and reliability of dietary information for both children and adults with IBS is poor ${ }^{98}$. In general, nutritional status and growth do not seem to be affected by these dietary manipulations; however, disordered eating (such as bulimia nervosa) might contribute to an increased incidence of obesity in children with IBS, as reported by one study ${ }^{99}$.

Despite the clinical experience of diet being associated with gastrointestinal symptoms, several issues exist in clearly defining causative foods. For example, children with FAPDs identify a wide variety of potential culprit foods ${ }^{96,100}$. However, when asked to rate symptom correlation (rarely, sometimes, often or always), children do not consistently identify the same foods and rate them as 'often' or 'always' causing their gastrointestinal symptoms, possibly explaining the high percentage of children with FAPDs consuming a potential culprit food in a 24-hour dietary recall ${ }^{95}$. 
Somatization

The manifestation of psychological distress in the form of physical symptoms, for which medical help is often sought.

\section{Catastrophizing}

A cognitive distortion that results in the person assuming that things are worse than they are or will have a far worse outcome than is realistic and often characterized by a lack of confidence and control.

Disease threat

An increased perception of the duration, frequency and seriousness of the symptoms.

Avoidance behaviours Any act or series of actions that enables an individual to anticipate or avoid unpleasant or painful situations, stimuli or events.

\section{Arthralgias}

Any discomfort or pain in the joints, although joint pain is not related to an inflammatory condition such as arthritis.

\section{Myalgias}

Muscle aches and pain, which can involve ligaments, tendons and fascia, that is, the soft tissues that connect muscles, bones and organs.
Malabsorption of carbohydrates can induce gastrointestinal symptoms. Malabsorption might be related to specific enzyme deficiencies (such as lactose in lactase deficiency; sucrose and starches in sucrase-isomaltase deficiency) or related to limited absorptive capacity for carbohydrates such as fructose, sorbitol or mannitol. In the case of smaller sugars, the osmotic load engendered can lead to small bowel luminal distention and rapid transit ${ }^{101}$. For larger sugars such as fructans, humans do not possess the enzymatic ability for digestion and, therefore, malabsorbed sugars are fermented by the colonic microbiota, leading to the formation of gas and potential colonic distention ${ }^{101}$.

\section{Psychological factors}

The physiology of the gut-brain axis is complex and includes afferent and efferent components. Signal transmission occurs via different pathways, including neural, hormonal and immunological components. The hypothalamic-pituitary-adrenal axis is hypothesized to be at the centre of gut-brain interactions owing to its important role in regulating psychological stress and mood, although the autonomic nervous system has also been found to play an important role ${ }^{102}$. The hypothalamic-pituitary-adrenal axis promotes gut-brain interactions via a feedback loop through the release of cortisol and corticotropin-releasing factor (both of which are increased in stressful conditions) as well as by stimulating mast cells and by inducing the release of pro-inflammatory cytokines ${ }^{102,103}$. Stress has been shown to increase gut inflammatory markers such as faecal calprotectin and CRP in both adults and paediatric patients with FAPDs ${ }^{102}$. Moreover, studies have reported that gut-brain interactions involve top-down and bottom-up processes and, therefore, gut microbiota can also influence brain function ${ }^{102-104}$. Under stressful conditions, gut microbiota can increase the permeability of the epithelial barrier, enabling antigens and/or pathogens to pass through and engendering an inflammatory reaction. The resultant circulating pro-inflammatory cytokines, such as IFN $\gamma$, IL- 1 and IL-6, might communicate with the central nervous system to stimulate an immune response in the brain, which may cause or exacerbate psychological symptoms (for example, anxiety and depression) ${ }^{105}$. A randomized controlled trial (RCT) found that probiotics can reduce depressive symptoms in adults with IBS and these improvements were associated with reduced reactivity of the limbic regions in the brain ${ }^{106}$. However, this RCT was a pilot study with a small sample size, making it difficult to draw any clear conclusions about the effectiveness of probiotic intervention. Whether the effect of probiotics is predominantly peripheral (intestinal) or central in nature is unknown and yet to be elucidated.

Several studies have shown increased psychological distress and behavioural problems in children with FAPDs compared with healthy children ${ }^{32,107}$. In contrast to adults, current evidence does not support the assumption that psychological symptoms such as depression and anxiety precede gut symptoms, such as abdominal pain, in children ${ }^{108}$. Psychological factors might be both a cause and a consequence of FAPDs, although psychological factors alone are not proven to be sufficient to cause FAPDs. Their aetiological role is hypothesized to be through interaction with other pathophysiological factors.

In addition, psychological factors are shown to have an impact on treatment outcomes once symptoms are already manifested. For example, in children with FAPDs, anxiety, depression, somatization (expressing multiple symptoms) and catastrophizing are associated with increased severity of symptoms, increased disability and disease persistence over several years ${ }^{32}$. These observational findings are supported by evidence from clinical trials showing that psychological interventions, such as cognitive behavioural therapy (CBT) and hypnotic therapy, are beneficial in reducing symptoms and disability as well as in increasing the quality of life ${ }^{109}$.

Despite the above-mentioned findings, the current literature reports that anxiety and depression might not be the most important factors in FAPD. Indeed, several studies found that specific pain-related cognitions and coping behaviours are directly related to pain and disability. For example, one study ${ }^{110}$ found that the effect of anxiety and depression on abdominal pain was mediated by somatization and pain catastrophizing. Studies on the active ingredients of CBT also found that treatment effects on disability and pain can be predicted by changes in pain-related cognitions such as catastrophizing, disease threat and avoidance behaviours ${ }^{111,112}$. This finding indicates the need to examine pain-specific psychological factors rather than anxiety and depression alone.

\section{Diagnosis, screening and prevention Patient history}

In most cases, children with FAPDs present with fairly stereotypical symptoms (BOX 1). Experienced medical providers frequently need little testing, if any, to make a prompt and a correct diagnosis. A detailed patient history and thorough physical examination are typically the necessary measures needed to establish FAPD diagnoses and to provide effective reassurance of the benign nature of the disease. The key elements in patient history include elucidating the characteristics of the pain, including location, quality, severity, duration, factors (especially food) that trigger or alleviate it, and its occurrence during sleep and excluding potential red flags such as involuntary weight loss or loose stools with blood. Clarifying whether symptom development was associated with a stressful event, for example, after an infection or traumatic episode such as the beginning of the school year, is particularly important. A detailed insight into the psychosocial history of both the child and the family is essential to uncover traits such as anxiety or catastrophizing, which are very prevalent in children with FAPDs and their parents. Typical hints that are indicative of a child with FAPD include the following: pain that is periumbilical and never goes away (that is, present for 24 hours every day); pain that does not consistently wake the child up at night but worsens under stress (for example, morning of school days); and co-occurrence of multiple somatic symptoms such as headaches, dizziness, arthralgias, myalgias and joint pain. Children with FAPDs may be as functionally disabled as children with organic diseases such as inflammatory bowel disease ${ }^{12}$. 
During the abdominal examination, evaluating whether distraction leads to a resolution of pain is important, a sign implying the presence of a FAPD. Performing the Carnett test (that is, evaluation of abdominal tenderness) is often helpful to differentiate abdominal wall pain syndrome from intra-abdominal pain originating from the viscera $^{113}$.

\section{Diagnosis}

The Rome criteria are often used to achieve a positive diagnosis $^{4}$ (FIG. 4). A child fulfilling a series of clinical criteria is eligible to receive a diagnosis of FAPD and can usually avoid any additional testing. The bottom of the Rome criteria for FAPDs includes the wording "after appropriate evaluation, the abdominal pain cannot be fully explained by another medical condition"; thus, the onus is on the physician to decide what an 'appropriate evaluation' might be. Evaluation certainly may include only history and a physical examination but the conception that this holds true in every case remains a platitude rather than a fact. Indeed, some blood and stool testing in children with a presumed FAPD is almost regularly done by the paediatric gastroenterologist (and consistently expected by parents); once referral is made to a subspecialist, the cost of care increases fivefold (excluding the cost of endoscopy) ${ }^{114}$.

Laboratory testing. Laboratory testing, when performed, needs to be targeted and limited to avoid overinterpretation of minor findings with no clinical significance. Serological testing for coeliac disease and measurement of faecal calprotectin seem to be the only

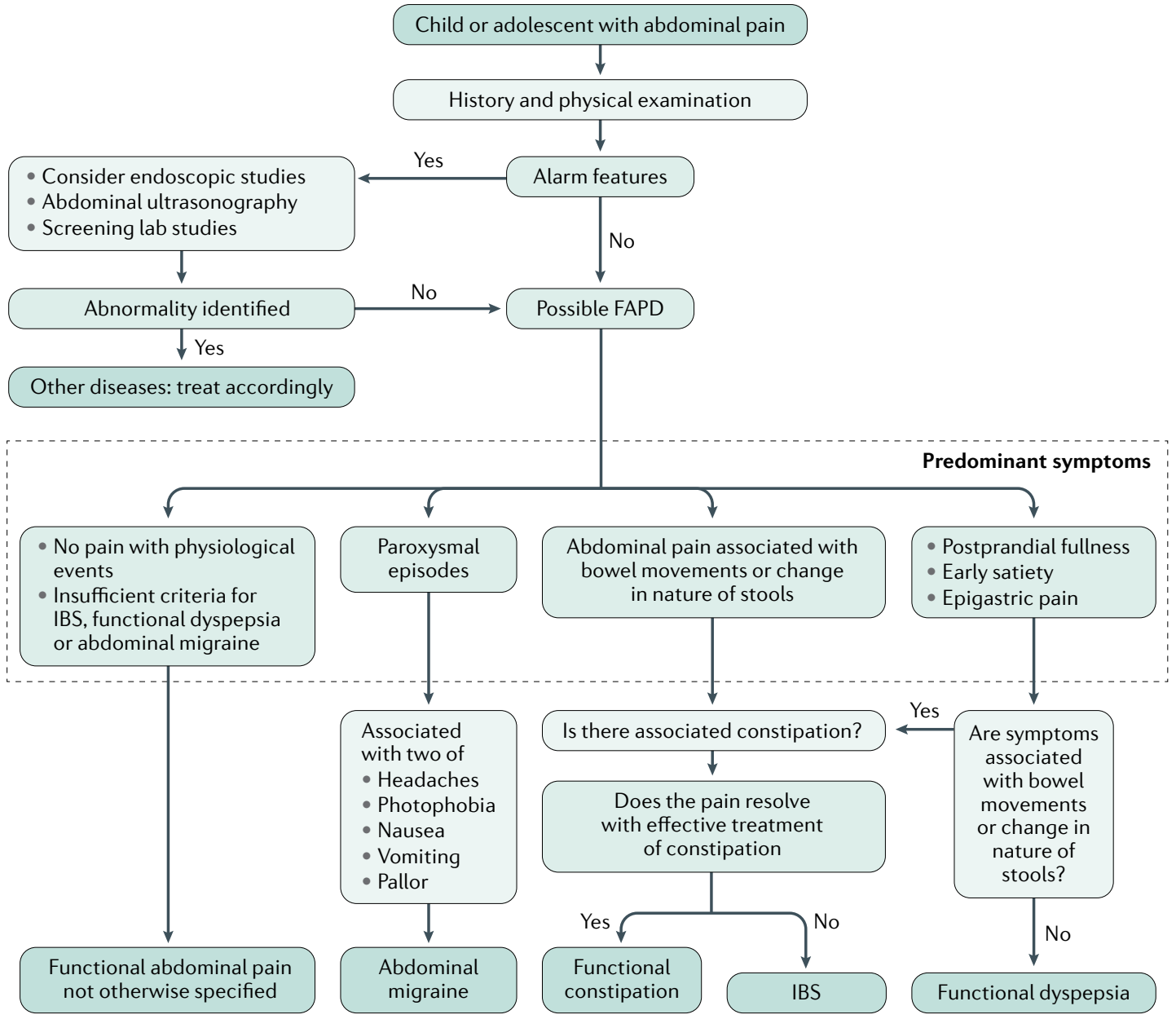

Fig. 4 | Rome IV diagnostic workflow of FAPDs. This figure illustrates the suggested algorithm for the diagnosis of a functional abdominal pain disorder (FAPD). Children presenting with symptoms of chronic or recurrent abdominal pain without an obvious organic pathology should undergo a careful clinical assessment (history and examination) to exclude alarm features. The patient history should include a detailed history from early life, family history and assessment of potential psychosocial factors. If an organic disorder is suspected, clinicians need to select appropriate investigations, which may include laboratory tests (for example, full blood count, serology testing for coeliac disease and faecal calprotectin), imaging (abdominal ultrasonography) and possibly endoscopy with mucosal assessment. In the absence of an obvious organic pathology, the clinician should consider the possibility and assess whether the symptom profile (using the Rome IV criteria) supports the diagnosis of one of the FAPDs (functional dyspepsia, irritable bowel syndrome (IBS), abdominal migraine and functional abdominal pain not otherwise specified). Notably, different types of FAPD may overlap in a single patient and with other functional gastrointestinal disorders such as 'functional constipation', which should be excluded before a diagnosis of IBS is made. Adapted with permission from REF. ${ }^{195}$, Copyright 2016 Rome Foundation, Inc. All Rights Reserved. 


\section{FODMAP}

Fermentable, Oligosaccharides, Disaccharides, Mono-

saccharides and Polyols, which are short-chain carbohydrates that are believed to be either

slowly absorbed or not

digested in the small intestine. cost-effective laboratory test in children with typical symptoms of FAPDs without alarm signs. An Italian study reported a $4 \%$ prevalence of coeliac disease in children fulfilling clinical diagnostic criteria for IBS ${ }^{115}$. However, one multinational, cross-sectional study failed to show a significant difference in the relative risk for FAPDs between patients with coeliac disease on a gluten-free diet and sibling controls without coeliac disease. Nevertheless, both groups showed a fourfold increase in the risk of FAPD compared with unrelated non-coeliac controls ${ }^{116}$. Thus, testing for coeliac disease by measuring serum tissue transglutaminase antibody and total IgA seems reasonable and should be considered, especially in children with an IBS-D phenotype. Evaluation of faecal calprotectin levels is being increasingly utilized for the non-invasive screening of intestinal mucosal inflammation and seems to be superior to standard serological testing such as complete blood count or CRP. Thus, in children with calprotectin levels $<50 \mu \mathrm{g} / \mathrm{g}$, endoscopy and histology add very little diagnostic value ${ }^{117}$. The role of Helicobacter pylori in children is controversial, although data suggest that Helicobacter pylori infections are not associated with FAPDs and its eradication does not correlate with improvement of abdominal pain ${ }^{118}$.

Endoscopy and imaging. The clinical value of gastrointestinal endoscopy in children with symptoms indicative of FAPD is limited. Minor macroscopic (for example, lymphonodular hyperplasia) and histopathological (increased mucosal mast cells and eosinophils) changes are frequently encountered on endoscopy and/or histology but lack clear clinical value. For example, emerging literature demonstrates the presence of inflammation with a predominance of mast cells and eosinophils in a proportion of children with functional dyspepsia.

Despite the common argument that a negative laboratory test leads to parental reassurance about the absence of an organic disease, evidence actually shows increased anxiety that something serious is missed ${ }^{119}$.

\section{Box 3 | Treatment options for functional dyspepsia}

The treatment of functional dyspepsia is multifaceted. Patient education is essential and all patients must be provided psychosocial support. Initially, dietary changes may be indicated, which may include smaller meals and avoidance of fatty foods and carbonated beverages. If the initial approach does not work ${ }^{240}$, then other modalities are tailored to the subtype of functional dyspepsia as outlined in the box below.

- For those with postprandial distress syndrome, which is believed to be associated with problems in gastric emptying and accommodation, cyproheptadine is recommended as one of the first-line drugs ${ }^{241,242}$. The latest use of a peppermint oil-based preparation has also been shown to be beneficial ${ }^{243-245}$. The next step in treatment is the use of prokinetics, including $5-\mathrm{HT}_{4}$ agonists, and when those fail, $5-\mathrm{HT}_{1}$ receptor agonists such as buspirone, which relax the proximal stomach, are recommended ${ }^{246}$. Antiemetics such as ondansetron, diphenhydramine, prochlorperazine and/or promethazine can be helpful to reduce nausea but can be sedating ${ }^{247}$. When all these treatments fail, neuromodulators in combination with cognitive behavioural therapy can be considered ${ }^{246,248}$.

- For those with epigastric distress syndrome, the use of antacids is usually the first-line therapy ${ }^{245,249}$. Similar to postprandial syndrome, the subsequent therapies are focused on altering stomach sensitivity and accommodation, with the use of cyproheptadine or herbal medications. If those fail, neuromodulation and psychological techniques are indicated.
Another study has shown that the prognosis is similar between children with FAPD who underwent a negative endoscopy and those who were not endoscopically tested $^{120}$.

Abdominal radiographs are often used to reveal 'occult constipation', which might be a source of abdominal pain, even in children who seem to have normal bowel habits. However, credible scientific evidence is lacking to prove that treatment with laxatives alleviates abdominal pain in a child who does not fulfil the Rome criteria for functional constipation. Indeed, when the aetiology of the pain is attributed to constipation, the evidence from the emergency department shows that these children are likely to experience a surgical outcome (for appendectomy) $)^{121}$.

Early diagnosis of FAPD in children increases the chances of a prompt resolution of symptoms ${ }^{122}$. Thus, the merits of any investigations that could potentially delay or distract from the diagnosis of a FAPD as well as the initiation of appropriate management should be carefully considered. Certainly, endoscopy should not be performed routinely for the diagnosis of FAPDs but only in cases where histological findings might support management options or in cases where differential diagnoses amenable to endoscopic (or histological) confirmation cannot be otherwise excluded.

\section{Management}

Regardless of the therapeutic approach, the importance of the patient-clinician relationship, effective communication and patient education at the outset cannot be emphasized enough, and these aspects remain at the heart of successful FAPD management. Ample time must be taken to explain the diagnosis to the carers of each individual patient as well as to discuss the biopsychosocial model (as it relates to each individual patient), subsequently designing a management plan, ensuring realistic expectations of prognosis and outcomes. We discuss the management with a particular focus on IBS and have highlighted the treatment options for functional dyspepsia (BOX 3).

The management of FAPDs is limited by several factors. First, owing to the biopsychosocial nature of the disorder, every child has a unique set of pathophysiological factors and responds differently to therapies (FIG. 5). In addition, the evidence base in children with FAPD is small and many treatment suggestions are therefore based on studies in adults. Nevertheless, as described below, children often do not respond similarly to adults. Even if a child would have access to all existing treatments, not all children respond adequately to current therapies. Finally, several efficacious treatments are behavioural (dietary and psychological) and are not readily available owing to a lack of allied health-care professionals as well as a lack of insurance coverage.

With the growing recognition of the role of gut microbiota, a number of interventions targeting gut microbiota, including probiotics, a low FODMAP diet, prebiotics and antibiotics, are increasingly being used for the management of FAPDs and several new therapies are on the horizon ${ }^{123}$. 


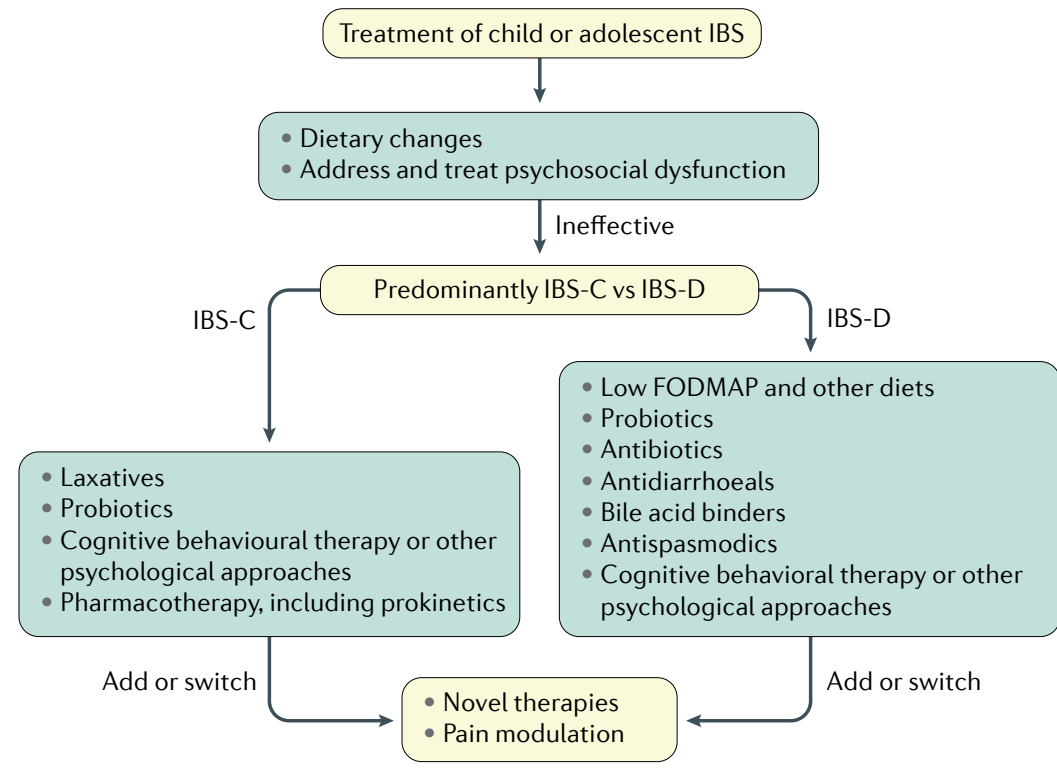

Fig. 5 | Therapeutic algorithm for IBS in children. Once a diagnosis of irritable bowel syndrome (IBS) is established, early engagement with psychosocial management is advised to develop a relationship for ongoing support and institution of the most effective therapeutic modality. Dietary interventions to optimize nutrition or help limit symptoms (for example, limited time trial of dietary exclusion if high possibility of food allergy is suspected or initiating low FODMAP diets) is recommended. Oversight of appropriate nutritional intake, preferably utilizing clinical dietitians or nutritionists, could also be undertaken. If these initial and non-invasive strategies are ineffective, the predominant type of IBS present, namely with constipation (IBS-C) or diarrhoea (IBS-D), must be determined. This information should then be used to design management strategies, although psychosocial management (such as behavioural interventions and hypnosis) should be utilized and maintained. Probiotics may also have some benefit in both the subtypes. The use of laxatives and/or prokinetics in IBS-C and of antibiotics, antidiarrhoeals or antispasmodics in IBS-D could be tested with appropriate review. For a select few patients, newer modalities such as neuromodulation may be beneficial. Adapted with permission from REF. ${ }^{195}$, Copyright 2016 Rome Foundation, Inc. All Rights Reserved.

Lactulose breath test

A test used to measure an increase in hydrogen and/or methane excreted in the breath, presumed to arise from the metabolism of ingested lactulose by intestinal bacteria. This test is used as a measure of small intestinal bacterial overgrowth or small bowel transit and to predict a patient's response to a low FODMAP diet. from a dietitian. Almost all published studies investigating the role of diet in the management of FAPDs involve IBS, with surprisingly little information on functional dyspepsia, abdominal migraine or FAP-NOS. For the management of functional dyspepsia, clinicians often promote small and frequent meals and low-fat foods, with little objective evidence showing benefit. Two systematic reviews on the use of diet to manage functional dyspepsia in adults acknowledged a lack of high-quality evidence to support dietary therapy in functional dyspepsia ${ }^{125,126}$.

Low FODMAP diet. Studies suggest that some patients with IBS are more sensitive to carbohydrate malabsorption than healthy individuals and, therefore, a low
FODMAP diet is usually also recommended for children with IBS. The rationale for the low FODMAP diet is a presumed reduction in microbial gas production and luminal distension, with a resultant decrease in pain ${ }^{127}$. Indeed, a few RCTs involving both children and adults have supported the use of low FODMAP diets or a specific reduction of fructans in the diet for a reduction of gastrointestinal symptoms ${ }^{88,128-130}$. However, current studies suggest that adult patients with IBS are more sensitive to FODMAP-induced luminal distension (rather than experiencing greater distension per se) than healthy individuals ${ }^{131}$, supporting the concept of visceral hypersensitivity. Furthermore, evidence also shows that FODMAP ingestion is associated with the generation of low-grade gut inflammation and barrier dysfunction $^{132,133}$.

However, a low FODMAP diet is complex and requires assistance from a dietitian ${ }^{134}$. A number of issues remain unresolved, including identifying which subset of patients will respond, the fact that the microbiome analysis of the stool is not practical as it requires standardized stool sampling and storage at home, and the predictive value of the lactulose breath test being controversial $^{88,129,135}$. In addition, the impact of removing potentially 'healthy' foods and the duration of the diet (response has been described within 2-7 days but longer durations of treatment are often recommended) are yet to be elucidated ${ }^{88,128}$. Furthermore, how and when eliminated foods should be reintroduced has to be determined as continuing a low FODMAP diet carries potential risks, which include a reduction in fibre, calcium and antioxidant intake and a decrease in some potentially beneficial gut bacteria (for example, Bifidobacteria spp. and Faecalibacterium prausnitzii) ${ }^{136}$.

Lactase deficiency seems to be less prevalent in children with FAPDs than in adult patients with IBS $^{137,138}$. Different polymorphisms in genes regulating sucrase-isomaltase activity seem to be more frequent in those with IBS-like symptoms than in the general population ${ }^{139}$. Molecular studies indicate a multitude of synthetic and trafficking defects that could affect the amount of functional enzyme produced, accounting for the wide variation in enzyme activity and thereby resulting in the diverse clinical phenotype ${ }^{140}$. Adults carrying polymorphisms associated with decreased sucrase-isomaltase activity might be less likely to respond to a low FODMAP diet ${ }^{141}$.
Gluten-free diet. Few studies have highlighted the potential role of non-IgE-mediated food allergy in causing IBS symptoms. Wheat, yeast, milk, soy and egg white were found to increase duodenal permeability and to cause lymphocytic and eosinophilic mucosal inflammation and clinical symptoms, predominantly abdominal pain in adults with IBS ${ }^{142,143}$. Of 108 patients with IBS, 61\% reacted to wheat. These food types are among the most common food allergens in the USA ${ }^{144}$. Future research is warranted to show whether such non-coeliac gluten hypersensitivity is of clinical importance in functional gastrointestinal disorders not only in adults but also in children. 
Prebiotics. Prebiotics can modify the gut microbial population and, presumably, the response to incompletely absorbed dietary components. Increasing the abundance of lactose-fermenting bacteria has been shown to improve symptoms of lactose-intolerance in adults with a history of self-reported milk intolerance ${ }^{145}$. A prebiotic ( $\beta$-galactooligosaccharide) was shown to be more effective in improving IBS symptoms than a low FODMAP diet in adults; the improvement of IBS symptoms (pain, distension and flatulence) outlasted the duration of prebiotic administration, whereas the response to the low FODMAP diet ended upon return to a normal $\operatorname{diet}^{146}$.

Enzyme and fibre supplementation. Enzyme supplementation has long been used to treat lactase deficiency and sucrase-isomaltase deficiency in children; however, in the latter deficiency, supplementation might not correct starch maldigestion ${ }^{147,148}$. Trials in adults have suggested that a similar strategy might be useful to treat malabsorption associated with certain FODMAP carbohydrates by improving their digestion and, in turn, reducing their availability to either exert an osmotic effect or to be fermented by bacteria, which are thought to distend the bowel and elicit IBS symptoms ${ }^{149,150}$. The use of enzyme supplementation may also allow some relaxation of the very restrictive low FODMAP diet and thereby improve compliance.

Insufficient dietary fibre intake in childhood has been associated with the development of FAPDs ${ }^{151,152}$. Meta-analyses of studies involving adults have shown benefits of soluble fibre in treating IBS $^{153,154}$. Following advice from studies in adults ${ }^{155}$, a randomized, double-blind controlled study in children reported a decrease in the frequency of abdominal pain in children with IBS treated with the soluble fibre psyllium compared with placebo (maltodextrin) ${ }^{156}$.

\section{Probiotics}

Multiple studies have examined the efficacy of probiotics (using a vast array of organisms) in the treatment of FAPDs, although the majority have been performed in adults with IBS. These investigations suggest that particular combinations of probiotics or specific species might be effective but their role remains unclear owing to study limitations such as sample size, blinding, differences in the probiotics used and the different dosing ${ }^{157}$. Although meta-analyses of paediatric trials have been performed, only few studies exist and with similar caveats to the adult meta-analyses ${ }^{158,159}$. Among five RCTs investigating children with FAP-NOS, the probiotic Lactobacillus reuteri DSM 1793 improved abdominal pain in three studies ${ }^{160-162}$, reduced functional disability but not abdominal pain in one study ${ }^{163}$, and was not superior to placebo in another ${ }^{164}$, although the methods of pain evaluation were different across studies. In children with IBS, two studies found that Lactobacillus rhamnosus GG was effective in reducing abdominal pain symptoms ${ }^{165,166}$ and one study reported no improvement in abdominal pain but an improvement in perceived abdominal distention; measures of pain differed yet again among the different studies. In one multicentre, crossover RCT performed in children with IBS, VSL\#3 (a mixture of eight probiotic strains) was found to be safe and more effective than placebo in ameliorating symptoms and in improving quality of life ${ }^{167}$.

\section{Psychological interventions}

Psychological interventions, such as CBT and hypnotherapy, have thus far proved to be the most successful interventions in managing FAPDs, arguing for their universal inclusion in the management strategies.

Cognitive behavioural therapy. CBT is the most studied psychological therapy for the treatment of IBS and it aims to alter the cognitions, emotions and behaviours, all of which might play a role in exacerbating or maintaining IBS symptoms. Studies in adults and children have shown that CBT is effective in improving pain and the ability to participate in social life as well as in alleviating symptoms of anxiety or depression compared with educational and other control interventions ${ }^{168-170}$. Twelve RCTs, involving 990 children (7-18 years of age) with IBS, showed the acute and long-lasting beneficial effects of CBT on quality of life, school performance and social participation. CBT can be provided as a face-to-face therapy $^{171-173}$ or targeted to children via the internet ${ }^{174}$ or to parents via the telephone ${ }^{175}$. However, the evidence is limited owing to the low quality of the trials. Owing to the limited access to comprehensive CBT programmes in most countries, internet-delivered CBT has the potential to increase the availability of treatment and reduce health-care costs ${ }^{176,177}$. Despite the small sample sizes and methodological weaknesses of these trials, the consistency and the magnitude of the reported effects of CBT prove its usefulness in improving pain and disability in children with IBS. Preliminary data suggest that internet-delivered CBT might also be useful for adolescents with functional dyspepsia ${ }^{112}$. Additionally, CBT focused only on parents is shown to be effective in reducing disability ${ }^{175}$. Although $\mathrm{CBT}$ has been used to treat migraine, evidence of its use in abdominal migraine or FAP-NOS is lacking. CBT in children with FAPDs is largely focused on learning to cope with the symptoms and reducing disability and, therefore, these therapies should ideally be part of an integrative care approach (for descriptions of such approaches see REF. ${ }^{109}$ and REF. ${ }^{178}$ ).

Hypnotherapy. In this approach, a patient is induced into a hypnotic state, guided by a therapist, to respond to suggestions to alter one's subjective experience, perception, emotion, thought or behaviour. In adults, hypnotherapy has been demonstrated to lead to changes in colonic motility, improve visceral hypersensitivity, and to reduce psychological factors such as somatization and psychological stress that persist long term ${ }^{179-181}$. However, in children with IBS, no reduction in hypersensitivity (tested experimentally) was found after hypnotherapy, although pain scores and somatization scores were lower than for children in standard medical care ${ }^{182}$. Five RCTs of children with IBS or FAP-NOS $(n=412$; 6-18 years of age), hypnotherapy, given either as individual therapy or as self-exercise at home using a $C D$, showed substantial long-lasting beneficial effects on quality of life, number of doctor visits and missed days 
of school (number needed to treat $=3)^{171,183}$. At the 1 -year and 5-year follow-up, $85 \%$ and $68 \%$, respectively, of patients receiving hypnotherapy were symptom free whereas only $25 \%$ and $20 \%$ of patients, respectively, were in remission in the control group ${ }^{184}$. Shortcomings of hypnotherapy include limited access, its rare coverage by commercial insurances and the lack of adequate welltrained hypnotherapists. As home-based hypnotherapy was non-inferior to therapist-based hypnotherapy, hypnotherapy using a CD might provide an attractive alternative treatment option for these children ${ }^{184}$.

Yoga therapy. Yoga practice has been shown to improve stress-induced underactivity of the parasympathetic nervous system. Three RCTs, including 127 children (7-18 years of age) with IBS, found positive effects of yoga therapy in decreasing abdominal pain compared with wait-list control or standard medical care ${ }^{171}$. However, a Dutch study showed that, at the 1-year follow-up, yoga intervention was not more effective than standard care that did not include $\mathrm{CBT}^{185}$. Owing to major flaws in study methods, a recommendation regarding yoga as a routine intervention for children with FAPDs cannot yet be made.

\section{Neurostimulation}

Several studies have shown the efficacy of electrical stimulation of the spinal cord and brain in modulating pain pathways ${ }^{186,187}$. This currently experimental technology was able to decrease the firing of $>50 \%$ of neurons in the spinal cord and central amygdala, thereby alleviating visceral pain. One study showed that percutaneous electrical nerve field stimulation (PENFS) modulated the response characteristics of neurons of the amygdala and the spinal cord and significantly reduced the development of visceral hypersensitivity in rats ${ }^{187}$. Subsequently, the same group showed, in a randomized, sham-controlled trial of 115 adolescents (11-18 years of age) with FAPDs, that PENFS with an active device improved well-being and resulted in a significant reduction in pain and disability compared with the sham-stimulated group ${ }^{188}$. Moreover, the beneficial effects of PENFS were sustained during the 2-month follow-up. Despite some ear discomfort, no serious adverse events were reported. Thus, PENFS seems to be a safe and effective approach and should be considered as a non-pharmacological alternative in children with FAPDs.

\section{Complementary and alternative medicine}

Complementary and alternative medicine includes different approaches and methodologies, ranging from acupuncture and ayurvedic medicine to chiropractic therapy, osteopathy, homeopathy, spiritual healing, massage and body-mind techniques like meditation. Approximately $40 \%$ of Australian and Dutch children diagnosed with FAPDs use one of these alternative therapies, with herbal remedies being the most common $(46 \%)^{189,190}$. Many of these complementary therapies are considered 'natural' by the general public and are therefore deemed safer and gentler than the armamentarium of modern medicine. Fear of the adverse effects associated with allopathic medication and the low perceived benefit of conventional treatment in children with FAPDs are potential reasons for parents to seek complementary and alternative therapies ${ }^{190}$. However, to date, RCTs evaluating the effect of herbal therapy, acupuncture, homeopathy, mind-body therapy or musculoskeletal manipulations, such as osteopathic and chiropractic manipulations, in children with FAPDs are not available $\mathrm{e}^{170}$.

Owing to the high spontaneous remission of FAPDs (30-70\%), a step-wise approach to management is judicious; education, identification and modification of stress factors and dietary interventions, if necessary, might be the first steps. When symptoms persist or reoccur, the next step could be initiating one of the psychological treatments, such as CBT and hypnotherapy, or PENFS.

\section{Pharmacological therapy}

Evidence supporting the efficacy of pharmacological treatments in children with FAPDs is lacking. In addition, the quality of the evidence is generally poor in existing studies, with only a few RCTs assessing the safety and efficacy of the most commonly used drugs (TABLE 1). The quality of these trials are criticized because of their small sample size, limited follow-up, inadequate concealment of allocation, lack of power and the use of non-validated questionnaires $^{191}$. The heterogeneity of study design, study outcomes, inclusion criteria and duration of treatments does not allow the pooling of data to perform a meta-analysis ${ }^{191}$. The treatment effects are also difficult to separate from the placebo response, which is generally known to be high in functional disorders ${ }^{192}$, higher in children than in adults ${ }^{193}$ and often driven by proxies parent reports of outcomes in RCTs show placebo effects as if they were the patients ${ }^{194}$.

The absence of conclusive data to support treatments based on scientific evidence and the lack of approved medications for the treatment of FAPDs in children challenge the physicians in daily practice. To fill this void, the Rome foundation has published the GI Genius Interactive Clinical Decision Toolkit ${ }^{195}$, which is a new, on-demand, online interactive software that combines the diagnostic and treatment decision algorithms to assist physicians in the management of patients with FAPDs. This toolkit was created through consensus of the Rome foundation board of directors and the Rome IV committee ${ }^{195}$. According to this online tool, the use of antispasmodics is recommended as the first-line treatment.

Antispasmodics. Only three RCTs have been conducted on the use of antispasmodics in children. A small, albeit positive, peppermint oil study (randomized double-blind, placebo-controlled trial) in children with IBS may be included in the list of RCTs evaluating antispasmodics ${ }^{196}$. Peppermint oil is assumed to act directly on intestinal smooth muscles or indirectly via receptor blockade on nerves of the intestinal smooth muscles to reduce intestinal contractions, which are deemed to manifest as spasms or cramps in patients with FAPDs. After 2 weeks, 75\% of children receiving peppermint oil reported reduced pain intensity compared with the placebo group, although 
Table 1 | Drug treatment trials in children with FAP

\begin{tabular}{|c|c|c|c|}
\hline Intervention & Inclusion criteria & Results & Ref. \\
\hline \multicolumn{4}{|c|}{ Antispasmodics } \\
\hline Trimebutine $^{a}$ & $\begin{array}{l}\text { Children and adolescents } \\
\text { ( } 4-18 \text { years of age), Rome III } \\
\text { criteria for IBS }\end{array}$ & $\begin{array}{l}\text { Clinical recovery (in pain or discomfort): } 94.9 \% \text { of children in } \\
\text { the treatment group, } 20.5 \% \text { in the control group }(P<0.0001)\end{array}$ & 198 \\
\hline Mebeverine ${ }^{b}$ & $\begin{array}{l}\text { Children with FAP } \\
\text { (6-18 years of age) }\end{array}$ & $\begin{array}{l}\text { Response rate (reduction in pain) on ITT analysis: } 40.6 \% \text { with } \\
\text { mebeverine, } 30.3 \% \text { with placebo }\end{array}$ & 199 \\
\hline Drotaverine $^{b}$ & $\begin{array}{l}\text { Children ( } 4-12 \text { years of age), } \\
\text { recurrent abdominal pain } \\
\text { (Apley criteria) }\end{array}$ & $\begin{array}{l}\text { Decrease in abdominal pain frequency }(P=0.01) \text {; decrease in } \\
\text { school absenteeism }(P=0.05)\end{array}$ & 200 \\
\hline \multicolumn{4}{|c|}{ Poorly absorbed antibiotics } \\
\hline Rifaximin $^{\mathrm{b}}$ & $\begin{array}{l}\text { Children ( } 3-15 \text { years of age), } \\
\text { Rome II criteria for IBS }\end{array}$ & $\begin{array}{l}\text { Benefit in improving abdominal pain, bloating and flatulence } \\
(P<0.005)\end{array}$ & 201 \\
\hline Rifaximin $^{\mathrm{b}}$ & $\begin{array}{l}\text { Children with recurrent } \\
\text { abdominal pain (8-18 years } \\
\text { of age), Rome II criteria }\end{array}$ & $\begin{array}{l}20 \% \text { of children treated with rifaximin (vs placebo) achieved } \\
\text { a normalized repeat LBT; no significant difference in } \\
\text { symptom improvement between groups }\end{array}$ & 202 \\
\hline \multicolumn{4}{|c|}{ Tricyclic antidepressant } \\
\hline Amitriptyline $^{\mathrm{b}}$ & $\begin{array}{l}\text { Adolescents with newly } \\
\text { diagnosed IBS ( } 12-18 \text { years } \\
\text { of age) }\end{array}$ & $\begin{array}{l}\text { Improvement in quality of life at weeks } 6,10 \text { and } 13(P=0.19 \text {, } \\
0.004 \text { and } 0.13 \text {, respectively); improvement in periumbilical } \\
\text { pain at week } 10(P=0.018)\end{array}$ & 203 \\
\hline Amitriptyline $^{c}$ & $\begin{array}{l}\text { Children with FAP, functional } \\
\text { dyspepsia and IBS ( } 8-17 \text { years } \\
\text { of age), Rome II criteria }\end{array}$ & $\begin{array}{l}\text { ITT analysis: in amitriptyline group, } 59 \% \text { of children reported } \\
\text { improvements in pain and anxiety; in placebo group, } 53 \% \text { of } \\
\text { children reported improvements in pain }(P=0.81)\end{array}$ & 204 \\
\hline \multicolumn{4}{|c|}{ SSRI antidepressant } \\
\hline Citalopram $^{\mathrm{d}}$ & $\begin{array}{l}\text { Children and adolescents } \\
\text { ( } 7-18 \text { years of age) with } \\
\text { recurrent abdominal pain }\end{array}$ & $\begin{array}{l}21 \text { patients; } 84 \% \text { were classified as responders (abdominal } \\
\text { pain, anxiety, depression, other somatic symptoms and } \\
\text { functional impairment), Clinical Global Impression } \\
\text { Scale-Improvement score } \leq 2\end{array}$ & 205 \\
\hline Citalopram $^{\mathrm{b}}$ & $\begin{array}{l}\text { Children with FAP } \\
\text { (6-18 years of age), } \\
\text { Rome Ill criteria }\end{array}$ & $\begin{array}{l}40.6 \% \text { of children responded (pain) to treatment, } 30.3 \% \\
\text { responded to placebo at } 4 \text { weeks }(P=0.17) ; 52.5 \% \text { of children } \\
\text { responded (pain) to treatment, } 41 \% \text { responded to placebo at } \\
8 \text { weeks ( } P=0.15 \text { ) }\end{array}$ & 206 \\
\hline
\end{tabular}

none of the antispasmodics routinely used in clinical practice in the USA was studied. Trimebutine is an opioid agonist that induces the release of motilin (which controls the pattern of smooth muscle cell contraction) and modulates the release of vasoactive intestinal peptide, glucagon and gastrin ${ }^{197}$. An RCT found trimebutine to be effective in relieving pain in children with IBS ${ }^{197,198}$ (TABLE 1). Additionally, a double-blind placebo-controlled trial in children with FAP-NOS showed significant benefit with the use of mebevirine (an anticholinergic agent that relaxes the intestinal smooth muscles and decreases peristalses) on pain compared with placebo ${ }^{199}$. A 4-week double-blind RCT investigating drotaverine (a non-cholinergic inhibitor) for the treatment of recurrent abdominal pain (Rome criteria were not applied) reported a significant decrease in abdominal pain frequency and absenteeism from school in children receiving drotaverine compared with placebo ${ }^{200}$.

Antibiotics. Rifaximin, a non-absorbable antibiotic, has been approved by the FDA for the treatment of IBS in adults ${ }^{94,197}$. An open trial showed that rifaximin significantly improved abdominal pain, bloating and flatulence in a group of 50 children with IBS with an abnormal lactulose breath hydrogen test compared with placebo ${ }^{201}$. Conversely, an RCT in children comparing rifaximin with placebo showed no benefit ${ }^{202}$ (TABLE 1). The lack of benefit was attributed to the low percentage of children treated with rifaximin who had normalization of the lactulose breath hydrogen test; therefore, more studies are required. Currently, routine use of rifaximin is not recommended for FAPDs and its use should be balanced against the potential adverse effects, including its potential interference with the establishment of a normal microbiome in young children.

Antidepressants. The Rome IV GI Genius Interactive Clinical Decision Toolkit recommends the use of antidepressants for patients who failed to improve with firstline treatment ${ }^{195}$. An RCT involving adolescents with IBS found benefit in overall quality of life in patients receiving amitriptyline for 13 weeks compared with placebo. However, the study found no improvement in abdominal pain in all quadrants and at all times, although periumbilical pain was reduced at week 10 out of 13 weeks treatment ${ }^{203}$. Furthermore, a large multicentre placebocontrolled RCT found no significant difference in global end points (such as quality of life and pain intensity) 
between amitriptyline and placebo after 4 weeks of treament ${ }^{204}$. However, anxiety scores were significantly improved in the amitriptyline group compared with placebo $^{204}$. An open label study conducted in 25 children and adolescents with recurrent abdominal pain (Rome criteria not employed) evaluating the efficacy and safety of citalopram (a selective serotonin reuptake inhibitor) showed a beneficial effect ${ }^{205}$, although a subsequent RCT in children with FAP-NOS failed to show significant benefit ${ }^{206}$. Currently, studies assessing the efficacy of antidepressants in other FAPDs are missing. Despite the lack of conclusive scientific evidence, antidepressants are commonly used in clinical practice to manage abdominal pain in children and adolescents. In 2004, the FDA issued a black-box warning following evidence suggesting that anti-depressants might be associated with increased suicidal thoughts and behaviours ${ }^{207}$. Although no data are available that show similar risks with the reduced dosing used for chronic pain treatment, this concern should be addressed with families when children are prescribed antidepressants. In addition, acquiring an electrocardiogram to screen for idiopathic long QT syndrome prior to antidepressant use owing to their potential to prolong the QT interval is commonly practised.

Analgesics and laxatives. Two small studies addressing the efficacy of gabapentin and pregabalin in adults with IBS $^{208,209}$ have demonstrated improvement in abdominal pain, urgency and bloating; however, no studies have been conducted in children with IBS so far.

Prucalopride (a 5- $\mathrm{HT}_{4}$ receptor agonist), lubiprostone (a prostaglandin $\mathrm{E}_{1}$ derivative) and linaclotide (a guanylate cyclase $\mathrm{C}$ agonist) have shown efficacy in the treatment of IBS-C in adults, although no trials have investigated these drugs in children with IBS ${ }^{85}$. However, RCTs in children with functional constipation did not show a significant benefit for prucalopride and lubiprostone compared with placebo ${ }^{210,211}$. As both drugs tend to accelerate transit, their use in IBS-D and in other FAPDs is not indicated.

The paucity of clinical trials, the lack of approved medications and the suboptimal outcomes of current treatments ${ }^{212}$ has prompted the Rome Foundation to publish recommendations for clinical trials for IBS in children $^{213}$. These recommendations were reviewed and discussed by a group of paediatric and adult gastroenterologists, psychologists, stakeholders, advocacy groups and members of the pharmaceutical industry at the GREAT 5 meeting in $2018\left(\right.$ REF. $\left.^{214}\right)$. The proceedings of this meeting are due to be published soon.

\section{Quality of life}

The severity and frequency of gastrointestinal symptoms have different impacts on the functional status of different patient groups. Patient-reported measures of health-related quality of life (HRQOL) should provide insight into the differential impact of FAPDs on the functional status of children and adolescents, including physical, emotional, behavioural, social and cognitive aspects $^{215}$.

Patient-reported outcomes that assess the impact of symptoms and HRQOL have become accepted measures of clinical status and treatment outcomes in adults. However, self-reported HRQOL measures in the paediatric setting have encountered challenges in instrument development and clinical application, in part owing to concerns about children's ability to reliably self-report health-related information.

A number of validated non-disease-specific (generic) HRQOL measures have been developed to measure the impact of paediatric diseases and treatment outcomes ${ }^{216}$ (BOX 4). Generic HRQOL measures provide patient metrics that can be compared with standard values established in healthy controls. In an effort to establish validated and calibrated HRQOL item banks in chronic diseases, the National Institutes of Health has launched the Patient Reported Outcomes Measurement Information System (PROMIS) ${ }^{217}$. The PROMIS Paediatric Cooperative Group developed paediatric self-report item banks for children (8-17 years of age) across five generic health domains (physical functioning, pain, fatigue, emotional health and social health) ${ }^{218}$. The PROMIS gastrointestinal symptom items have been developed and validated in adults but are yet to be validated in paediatric patients ${ }^{219}$.

HRQOL of paediatric patients with FAPDs can be assessed using generic and/or disease-specific questionnaires. The Paediatric Quality of Life Inventory 4.0 contains both generic core scales and disease-specific modules $^{220}$. The generic core has been validated and is used to compare healthy children and children with various disorders ${ }^{221-223}$. The multidimensional PedsQL Gastrointestinal Symptoms Scale provides a common metric across both functional and organic gastrointestinal diseases and includes parallel child self-report and parent proxy-report formats (for children 5-18 years of age) and proxy reports from parents (for children 2-4 years of age). The scale assesses stomach pain and stomach discomfort during food and drink intake, trouble swallowing, heartburn and reflux, nausea and vomiting, gas and bloating, constipation, blood in stool and diarrhoea. The Gastrointestinal Symptoms Scale has been well validated in functional and organic gastrointestinal diseases and standardized to healthy controls ${ }^{224-226}$.

Quantifying individual gastrointestinal symptoms are important to develop patient-centred and symptom-targeted interventions, which can ameliorate the overall HRQOL. For example, one multicentre study of 259 paediatric patients with functional constipation, functional abdominal pain or IBS found that individual gastrointestinal symptoms best predicted impaired overall HRQOL ${ }^{217}$.

Using the PedsQL 4.0 Generic Core Scale, a key study compared HRQOL in paediatric patients with FAPD and in children with organic gastrointestinal disease with a sample of healthy controls matched for sex, age and ethnicity ${ }^{227}$. The sample included 689 families of patients with functional and organic gastrointestinal disorders and 1,114 healthy control families. Patients with FAPDs and organic gastrointestinal disease had decreased HRQOL compared with healthy controls. Paradoxically, children with FAPDs demonstrated lower generic HRQOL scores in all dimensions compared with children with organic disease. This finding might 


\section{Box $4 \mid$ HRQOL questionnaires for children}

- PedsQL - This is a modular approach measuring HRQOL in healthy and diseased children and adolescents in four domains (physical, emotional, social and school functioning) and three summary scores (total score as well as physical and psychosocial health scores) ${ }^{22}$

- KIDSCREEN - This is a HRQOL questionnaire for children and adolescents (8-18 years of age) that evaluates children's and adolescents' subjective health and well-being ${ }^{250}$

- KINDL - This is a questionnaire-based generic instrument for assessing HRQOL in children and adolescents 3 years of age and older ${ }^{251}$

- GCQ (Generic Children's QOL) - This instrument is used to measure the quality of life in children 6-14 years of age; the GCQ is not symptom-oriented or problem-specific but focuses on areas of interest for all children such as families, peers and school ${ }^{252}$

- HUI (Health Utilities Index) - This tool classifies the health domains into sensation, mobility, pain, cognition, ambulation and emotion. HUI values are a common component of the quality-adjusted life years calculations used in population health and economics ${ }^{25}$

- ITQOL (Infant Toddler QOL Questionnaire) - This questionnaire adopts the WHO definition of health and was developed for use in infants and toddlers from 2 months to 5 years of age ${ }^{254}$

- TAPQOL (TNO-AZL Questionnaire for Preschool Children's Health-Related Quality of Life) - This instrument was developed to meet the need for a reliable and valid instrument for measuring parental perceptions of $\mathrm{HRQOL}$ in preschool children ${ }^{25}$

- TACQOL (TNO-AZL Questionnaire for Children's Health-Related Quality of Life)This questionnaire allows a description of $\mathrm{HRQOL}$ of children (6-15 years of age) with chronic diseases via self-reporting or proxy-reporting by parents ${ }^{25}$

- PROMIS (Patient-Reported Outcomes Measurement Information System) - This tool is a set of patient-centred measures that evaluates and monitors physical, mental and social health in healthy individuals and in adults and children with functional disorders ${ }^{25}$

HRQOL, health-related quality of life.

be explained by patient perception of control over the disease process and response shifts on the metric ${ }^{228}$. An important part of quality of life and an aim of FAPD treatment is disability ${ }^{213}$. Disability in children with FAPD includes school absences, not participating in sports or other peer activities, and avoiding chores. The Functional Disability Inventory is the most widely used scale in research and clinical care to measure disability in $\mathrm{FAPD}^{229,230}$. The Functional Disability Inventory assesses activity limitations in daily life that have been shown to be responsive to treatment ${ }^{170}$.

Assessment of patient-reported symptoms and HRQOL can provide clinical options for families and children to increase their perceived control over symptoms and encourage appropriate behavioural changes in an effort to improve the quality of life of children ${ }^{228}$.

\section{Outlook}

FAPDs are common in childhood and present the archetypal functional pain disorder with their biopsychosocial paradigm. FAPDs are likely universal and potentially increasing in prevalence and/or complexity. Therefore, well-designed epidemiological studies from across all geographical regions as well as cultural and ethnic communities across the world are needed to truly understand the nature and the size of the problem.

\section{Mechanisms}

Of specific importance is our current understanding of the potential factors that contribute to the aetiopathogenesis of FAPDs in children. Basic and translational science research addressing immune dysfunction and nerve dysfunction using tissue samples from paediatric patients with FAPDs are required. As noted above, early-life programming ${ }^{42}$, that is, events, generally insults or traumas (psychosocial or medical), occurring at critical stages of development of the various elements of the complex gut-brain axis, has been shown to predispose susceptible individuals to develop FAPDs. Whether this bears any relevance in the development of FAPDs in adults is unclear, although there is reason to believe this might be the case. Early-life history is probably not often sought or might be overlooked in these patients. Whether childhood FAPDs develop into the same or different functional gastrointestinal disorders in adult life is uncertain. Although current evidence suggests that children with FAPDs are more likely to develop psychological or psychiatric morbidities, long-term cohort studies are warranted to address these questions $\mathrm{s}^{231-233}$.

Factors that aberrantly alter gut microbiota may alter the structural or functional integrity of the enteric nervous system and, in turn, their intricate and carefully balanced interactions with the central nervous system. The consequence may be a reprogramming of the gastrointestinal tract towards a hypersensitive state and of the brain towards a hypervigilant state. Of course, other genetic factors, social factors and physiological changes, such as those occurring at puberty or adolescence, might also contribute to symptom development. Currently, the influences of 'modern age', such as digital or social media, omnipresent electronic gadgets and social changes, imparted by these factors are yet to be elucidated. These modern tools are increasingly associated with anxiety and stress ${ }^{234}$ and might therefore potentially have a substantial impact on FAPDs. A deeper understanding of all these influences and their timing in relation to triggering the eventual development of FAPDs may facilitate much-needed, enhanced preventive or therapeutic strategies.

\section{Diagnosis}

The diagnosis of FAPDs remains a challenge. By the time children are investigated, the early-life insults may have already occurred and changes in pain sensitivity and/or processing might have been established despite the absence of obvious pathology. To date, consensus on the type of investigations, if any, or of the age at which children should be investigated are not available. Clinical evaluation remains the essential mainstay and presently, outside of research, there is little place for routine investigations, especially those that are more invasive.

\section{Management}

The long-term outcomes of therapies for paediatric FAPD, such as hypnotherapy, are still being established, with up to $\sim 40 \%$ of children continuing to have symptoms despite treatment ${ }^{235,236}$. Certainly, negative prognostic factors, such as chronicity of the FAPD, presence of nausea and/or extraintestinal symptoms, positive family history of FAPDs, and a failure to engage with appropriate therapies or therapists, play a part ${ }^{231,232,237-239}$. Further clarification of the negative prognostic factors that allow the targeting of patients most at need for 
intervention as well as better definition and development of optimal and accessible management are required. To facilitate such a stratification, establishing and validating the most appropriate primary outcome measures and specific tools for the assessment of symptoms and response to therapy in the paediatric population remain a prerequisite.

The use pharmacological therapy for the management of FAPDs remains disappointing. Future studies should focus on understanding the factors underlying the magnitude of placebo effects and how these can be minimized in drug trials or harnessed during therapy. Current data, albeit preliminary, suggests that manipulating the gut microbiome at critical time points has potential therapeutic value but this needs further exploration, especially given that no definitive role for probiotics has been indentified ${ }^{157}$. Despite clear evidence of the advantage and efficacy of psychological therapies and social interventions for the management of FAPDs, the authors of the studies acknowledge considerable variability and the availability of these interventions outside large referral centres. The efficacy of psychological therapies needs to be specifically explored in well-designed studies and addressed in the community and across diverse geographical settings. Interestingly, despite their potential contribution to pathogenesis, newer 'digital' technologies might actually be beneficially utilized to address challenges in health care and facilitate the delivery of therapy 'at home'. Future studies should address this avenue, especially given increased limitations on the availability of face-to-face therapies, highlighted by the global coronavirus pandemic. Unfortunately, a number of poor-quality applications claim to be CBT but have not been validated, highlighting the importance of ensuring content expertise as well as an evidence basis for online or digital therapies. Nonetheless, the urgent need for supporting, effective psychosocial interventions for the management of FAPDs should be considered by governmental agencies and health-care organizations as well as by policymakers working in conjunction with physicians.
Although subdividing FAPDs into distinct disorders is essential, further research into the identification of biological signatures (biomarkers) to compliment or supersede symptom-based phenotyping or even to further define the conditions is critical to progressing the field. Perhaps one of the greatest challenges continues to be the use and abuse of the umbrella term 'functional'. This term was meant to indicate that the gut, albeit healthy, functions differently. However, this term is often misinterpreted as 'fictional' by so many primary care physicians, paediatricians and gastroenterologists alike and seems to reinforce a rather mystical view of the entity of FAPDs. This mix-up is especially challenging given that many FAPDs result from or coexist with distinct organic pathologies such as coeliac disease and inflammatory bowel disease. From our experience, patients and their families often describe a history of battling insinuations of malingering or fabrication. In acknowledgement of distinct, albeit subtle, pathologies underlying what can be devastating disorders as described in this Primer, the Rome committee has recommended functional gastrointestinal disorders, of which FAPDs are a subset of, to be renamed as 'disorders of gut-brain interaction'. Whether this renaming will remove the associated stigma remains to be ascertained. The stigma associated with these disorders is not due to a confusion of the term; other terminologies prior to 'functional' were also stigmatizing, including unexplained or psychosomatic disorders. If physicians continue focusing on biological causes as the only valid reason for symptoms, disorders of gut-brain interactions will soon be perceived as invalid and 'just in one's head'. Focusing solely on biological causes is a disservice not only to this patient population but to all diseases and disorders.

Overall, FAPDs in children have seen considerable progress in the last decade, with the hope that this will herald tangible steps towards harmonizing approaches to classify, diagnose and manage these challenging, often perplexing, conditions.

Published online: 05 November 2020
1. Robin, S. G. et al. Prevalence of pediatric functional gastrointestinal disorders utilizing the Rome IV criteria. J. Pediatr. 195, 134-139 (2018).

A large study collecting data on the prevalence of functional gastrointestinal disorders from families from the 50 states of the USA.

2. Drossman, D. A. Functional gastrointestinal disorders: history, pathophysiology, clinical features and Rome IV Gastroenterology 150, 1262-1279 (2016).

3. Benninga, M. A et al. Childhood functional gastrointestinal disorders: neonate/toddler. Gastroenterology https://doi.org/10.1053/ j.gastro.2016.02.016 (2016).

4. Hyams, J. S. et al. Functional disorders: children and adolescents. Gastroenterology https://doi.org/ 10.1053/j.gastro.2016.02.015 (2016). This paper finally brought together the different definitions of functional disorders in children under one umbrella definition (Rome IV).

5. Rajindrajith, S. \& Devanarayana, N. M. Subtypes and symptomatology of irritable bowel syndrome in children and adolescents: a school-based survey using Rome III criteria. J. Neurogastroenterol. Motil. 18, 298-304 (2012).

6. Turco, R. et al. Do distinct functional dyspepsia subtypes exist in children? J. Pediatr. Gastroenterol. Nutr. 62, 387-392 (2016).

7. Schurman, J. V. et al. Diagnostic utility of the water load test in children with chronic abdomina pain. J. Pediatr. Gastroenterol. Nutr. 44, 51-57 (2007).

8. Edwards, T., Friesen, C. \& Schurman, J. V. Classification of pediatric functional gastrointestinal disorders related to abdominal pain using Rome III vs. Rome IV criterions. BMC Gastroenterol. 18, 41 (2018).

9. Rasquin, A. et al. Childhood functional gastrointestinal disorders: child/adolescent. Gastroenterology 130. 1527-1537 (2006).

10. Diederen, K. et al. The prevalence of irritable bowel syndrome-type symptoms in paediatric inflammatory bowel disease, and the relationship with biochemical markers of disease activity. Aliment. Pharmacol. Ther. 44, 181-188 (2016)

11. Watson, K. L. Jr, Kim, S. C., Boyle, B. M. \& Saps, M Prevalence and impact of functional abdominal pain disorders in children with inflammatory bowel diseases (IBD-FAPD). J. Pediatr. Gastroenterol. Nutr. 65, 212-217 (2017)

12. Youssef, N. N., Murphy, T. G., Langseder, A. L. $\&$ Rosh, J. R. Quality of life for children with functional abdominal pain: a comparison study of patients' and parents' perceptions. Pediatrics 117, 54-59 (2006).

13. Varni, J. W. et al. Health-related quality of life in pediatric patients with irritable bowel syndrome: a comparative analysis. J. Dev. Behav. Pediatr. 27 , 451-458 (2006).
14. Rasquin-Weber, A. et al. Childhood functiona gastrointestinal disorders. Gut 45 (Suppl. 2), II60-II68 (1999).

15. Saps, M. \& Di Lorenzo, C. Interobserver and intraobserver reliability of the Rome II criteria in children. Am. J. Gastroenterol. 100, 2079-2082 (2005).

16. Chogle, A., Dhroove, G., Sztainberg, M., Di Lorenzo, C $\&$ Saps, M. How reliable are the Rome III criteria for the assessment of functional gastrointestinal disorders in children? Am. J. Gastroenterol. 105, 2697-2701 (2010).

17. Chogle, A. et al. Accuracy of pain recall in children. J. Pediatr. Gastroenterol. Nutr. 55, 288-291 (2012).

18. van der Plas, R. N., Benninga, M. A., Redekop, W. K., Taminiau, J. A. \& Büller, H. A. How accurate is the recall of bowel habits in children with defaecation disorders? Eur. J. Pediatr. 156, 178-181 (1997).

19. Koppen, I. J. N., Velasco-Benitez, C. A., Benninga, M. A., Di Lorenzo, C. \& Saps, M. Using the Bristol stool scale and parental report of stool consistency as part of the Rome III criteria for functional constipation in infants and toddlers. J. Pediatr. 177, 44-48.e41 (2016).

20. Sjölund, J. et al. Prevalence and progression of recurrent abdominal pain, from early childhood to adolescence. Clin. Gastroenterol. Hepatol. https://doi. org/10.1016/j.cgh.2020.04.047 (2020). 
21. Saps, M., Velasco-Benitez, C. A., Langshaw, A. H. \& Ramirez-Hernandez, C. R. Prevalence of functional gastrointestinal disorders in children and adolescents: comparison between Rome III and Rome IV criteria. J. Pediatr. 199, 212-216 (2018).

22. Devanarayana, N. M., Adhikari, C., Pannala, W. $\Sigma$ Rajindrajith, S. Prevalence of functional gastrointestinal diseases in a cohort of Sri Lankan adolescents: comparison between Rome II and Rome III criteria. J. Trop. Pediatr. 57, 34-39 (2011).

23. Korterink, J. J., Diederen, K., Benninga, M. A. \& Tabbers, M. M. Epidemiology of pediatric functional abdominal pain disorders: a meta-analysis. PLOS ONE 10, e0126982 (2015)

This paper is the best worldwide survey of prevalence or incidence of functional bowe disorder in children and adolescents, irrespective of the underlying definitions and using a large variety of methods

24. Lewis, M. L., Palsson, O. S., Whitehead, W. E. $\Sigma$ van Tilburg, M. A. L. Prevalence of functiona gastrointestinal disorders in children and adolescents. J. Pediatr. 177, 39-43.e3 (2016).

25. Udoh, E., Devanarayana, N. M., Rajindrajith, S., Meremikwu, M. \& Benninga, M. A. Abdominal painpredominant functional gastrointestinal disorders in adolescent Nigerians. J. Pediatr. Gastroenterol. Nutr 62, 588-593 (2016)

26. Gulewitsch, M. D., Enck, P., Schwille-Kiuntke, J., Weimer, K. \& Schlarb, A. A. Rome III criteria in parents' hands: pain-related functional gastrointestinal disorders in community children and associations with somatic complaints and mental health. Eur. $J$. Gastroenterol. Hepatol. 25, 1223-1229 (2013).

27. Sagawa, T. et al. Functional gastrointestinal disorders in adolescents and quality of school life. J. Gastroenterol. Hepatol. 28, 285-290 (2013).

28. Adeniyi, O. F., Lesi, O. A., Olatona, F. A., Esezobor, C. I. $\&$ Ikobah, J. M. Irritable bowel syndrome in adolescents in Lagos. Pan Afr. Med. J. 28, 93 (2017)

29. Carson, L. et al. Abdominal migraine: an underdiagnosed cause of recurrent abdominal pain in children. Headache 51, 707-712 (2011).

30. Mortimer, M. J., Kay, J. \& Jaron, A. Clinical epidemiology of childhood abdominal migraine in an urban general practice. Dev. Med. Child Neurol. 35, 243-248 (1993)

31. Dahl-Larsen, R., Buhl, S. B., Husby, S. \& Qvist, N. Recurrent abdominal pain, dyspepsia and constipation in children aged 9-13. A questionnaire investigation[Danish]. Ugeskr. Laeger 167 1848-1851 (2005)

32. Newton, E., Schosheim, A., Patel, S., Chitkara, D. K. \& van Tilburg, M. A. L. The role of psychological factors in pediatric functional abdominal pain disorders. Neurogastroenterol. Motil. 31, e13538 (2019).

33. Zernikow, B. et al. Characteristics of highly impaired children with severe chronic pain: a 5-year retrospective study on 2249 pediatric pain patients. BMC Pediatr. 12, 54 (2012)

34. Levy, R. L. et al. Increased somatic complaints and health-care utilization in children: effects of parent IBS status and parent response to gastrointestinal symptoms. Am. J. Gastroenterol. 99, 2442-2451 (2004).

35. Sherman, A. L., Bruehl, S., Smith, C. A. \& Walker, L. S Individual and additive effects of mothers' and fathers' chronic pain on health outcomes in young adults with a childhood history of functional abdominal pain. J. Pediatr. Psychol. 38, 365-375 (2013).

36. Levy, R. L. Exploring the intergenerational transmission of illness behavior: from observations to experimental intervention. Ann. Behav. Med. 41, 174-182 (2011).

37. Stone, A. L., Bruehl, S., Smith, C. A., Garber, J. \& Walker, L. S. Social learning pathways in the relation between parental chronic pain and daily pain severity and functional impairment in adolescents with functional abdominal pain. Pain 159, 298-305 (2018).

38. Craig, K. D. Social communication model of pain. Pain 156, 1198-1199 (2015).

39. Van Der Veek, S. M. et al. Do parents maintain or exacerbate pediatric functional abdominal pain? A systematic review and meta-analysis. J. Health Psychol. 17, 258-272 (2012).

40. Dong, Y.-Y. Prevalence of irritable bowel syndrome in Chinese college and university students assessed using Rome III criteria. World J. Gastroenterol. 16, 4221 (2010)

41. Son, Y. J., Jun, E. Y. \& Park, J. H. Prevalence and risk factors of irritable bowel syndrome in Korean adolescent girls: a school-based study. Int. J. Nurs. Stud. 46, 76-84 (2009).

42. Bonilla, S. \& Saps, M. Early life events predispose the onset of childhood functional gastrointestinal disorders. Rev. Gastroenterol. Mex. 78, 82-91 (2013).

43. Levy, R. L. et al. Irritable bowel syndrome in twins: heredity and social learning both contribute to etiology. Gastroenterology 121, 799-804 (2001).

44. van Tilburg, M. A., Zaki, E. A., Venkatesan, T. \& Boles, R. G. Irritable bowel syndrome may be associated with maternal inheritance and mitochondrial DNA control region sequence variants. Dig. Dis. Sci. 59, 1392-1397 (2014).

45. Faure, C. \& Grunder, F. R. in Pediatric Neurogastroenterology (eds Faure, C.; Thapar, N. \& Di Lorenzo, C.) 39-52 (Springer, 2017).

46. Halac, U., Noble, A. \& Faure, C. Rectal sensory threshold for pain is a diagnostic marker of irritable bowel syndrome and functional abdominal pain in children. J. Pediatr. 156, 60-65.e1 (2010).

47. Woolf, C. J. Central sensitization: implications for the diagnosis and treatment of pain. Pain 152, S2-S15 (2011).

48. Pas, R. et al. Hyperexcitability of the central nervous system in children with chronic pain: a systematic review. Pain Med. 19, 2504-2514 (2018).

49. Sherman, A. L., Morris, M. C., Bruehl, S. Westbrook, T. D. \& Walker, L. S. Heightened temporal summation of pain in patients with functional gastrointestinal disorders and history of trauma. Ann. Behav Med 49, 785-792 (2015).

50. Liu, X. et al. Excessive coupling of the salience network with intrinsic neurocognitive brain networks during rectal distension in adolescents with irritable bowel syndrome: a preliminary report. Neurogastroenterol. Motil. 28, 43-53 (2016).

51. Langley-Evans, S. C. Nutrition in early life and the programming of adult disease: a review. J. Hum Nutr. Diet. 28 (Suppl. 1), 1-14 (2015)

52. Rosen, J. M., Adams, P. N. \& Saps, M. Umbilical hernia repair increases the rate of functional gastrointestinal disorders in children. J. Pediatr. 163, 1065-1068 (2013)

53. Saps, M. \& Bonilla, S. Early life events: infants with pyloric stenosis have a higher risk of developing chronic abdominal pain in childhood. J. Pediatr. 159 551-554.e1 (2011).

54. Saps, M. et al. Post-infectious functional gastrointestinal disorders in children. J. Pediatr. 152, 812-816 (2008)

55. Thabane, M. et al. An outbreak of acute bacterial gastroenteritis is associated with an increased incidence of irritable bowel syndrome in children. Am. J. Gastroenterol. 105, 933-939 (2010).

56. Cremon, C. et al. Salmonella gastroenteritis during childhood is a risk factor for irritable bowel syndrome in adulthood. Gastroenterology 147, 69-77 (2014). This study documents the existence of postinfection IBS in adults after an exposure to a bacteria (Salmonella) during childhood, nearly 25 years before the outbreak, although exposed adults did not report higher incidence of IBS than controls without such exposure.

57. Saps, M., Adams, P., Bonilla, S., Chogle, A. \& Nichols-Vinueza, D. Parental report of abdominal pain and abdominal pain-related functional gastrointestinal disorders from a community survey. J. Pediatr. Gastroenterol. Nutr. 55, 707-710 (2012).

58. Saps, M., Dhroove, G. \& Chogle, A. Henoch-Schonlein purpura leads to functional gastrointestinal disorders. Dig. Dis. Sci. 56, 1789-1793 (2011).

59. Anand, K. J., Runeson, B. \& Jacobson, B. Gastric suction at birth associated with long-term risk for functional intestinal disorders in later life. J. Pediatr. 144, 449-454 (2004)

60. Saps, M. Lu, P \& Bonilla, S. Cow's-milk allergy is a risk factor for the development of FGIDs in children. J. Pediatr. Gastroenterol. Nutr. 52, 166-169 (2011).

61. Uusijarvi, A. et al. Use of antibiotics in infancy and childhood and risk of recurrent abdominal pain - a Swedish birth cohort study. Neurogastroenterol. Motil. 26, 841-850 (2014).

62. Barreau, F., Salvador-Cartier, C., Houdeau, E. Bueno, L. \& Fioramonti, J. Long-term alterations of colonic nerve-mast cell interactions induced by neonatal maternal deprivation in rats. Gut 57 , 582-590 (2008).

63. Nurgali, K. et al. Morphological and functional changes in guinea-pig neurons projecting to the ilea mucosa at early stages after inflammatory damage. J. Physiol. 589, 325-339 (2011).
64. Collins, J., Borojevic, R., Verdu, E. F., Huizinga, J. D. \& Ratcliffe, E. M. Intestinal microbiota influence the early postnatal development of the enteric nervous system. Neurogastroenterol. Motil. 26, 98-107 (2014).

65. Di Nardo, G. et al. Neuroimmune interactions at different intestinal sites are related to abdominal pain symptoms in children with IBS. Neurogastroenterol. Motil. 26, 196-204 (2014).

66. Friesen, C. A. et al. Antral inflammatory cells, gastric emptying, and electrogastrography in pediatric functional dyspepsia. Dig. Dis. Sci. 53, 2634-2640 (2008).

67. Wauters, L., Nightingale, S., Talley, N. J., Sulaiman, B. $\&$ Walker, M. M. Functional dyspepsia is associated with duodenal eosinophilia in an Australian paediatric cohort. Aliment. Pharmacol. Ther. 45, 1358-1364 (2017).

68. Schappi, M. G. et al. Mast cell-nerve interactions in children with functional dyspepsia. J. Pediatr. Gastroenterol. Nutr. 47, 472-480 (2008).

69. Karunanayake, A., Rajindrajith, S., de Silva, H. A., Gunawardena, S. \& Devanarayana, N. M. Autonomic functions and gastric motility in children with functional abdominal pain disorders. World J. Gastroenterol. 25, 95-106 (2019).

70. Friesen, C. A. et al. Electrogastrography in pediatric functional dyspepsia: relationship to gastric emptying and symptom severity. J. Pediatr. Gastroenterol. Nutr. 42, 265-269 (2006)

71. Vargas-Luna, F. M. et al. Electrogastrographic and autonomic nervous system responses to solid and liquid meals in youth with functional dyspepsia. Neurogastroenterol. Motil. 32, e13785 (2020).

72. Jericho, H., Adams, P., Zhang, G., Rychlik, K. \& Saps, M. Nausea predicts delayed gastric emptying in children. J. Pediatr. 164, 89-92 (2014).

73. Riezzo, G., Russo, F. \& Indrio, F. Electrogastrography in adults and children: the strength, pitfalls, and clinical significance of the cutaneous recording of the gastric electrical activity. Biomed. Res. Int. 2013. 282757 (2013)

74. Hoffman, I. \& Tack, J. Assessment of gastric motor function in childhood functional dyspepsia and obesity. Neurogastroenterol. Motil. 24, 108-112, e81 (2012)

75. Di Lorenzo, C. et al. Visceral hyperalgesia in children with functional abdominal pain. J. Pediatr. 139 838-843 (2001)

76. Kovacic, K. \& Di Lorenzo, C. Functional nausea in children. J. Pediatr. Gastroenterol. Nutr. 62, 365-371 (2016).

77. Wang, X. J. \& Camilleri, M. Personalized medicine in functional gastrointestinal disorders: understanding pathogenesis to increase diagnostic and treatment efficacy. World J. Gastroenterol. 25, 1185-1196 (2019).

78. DuPont, A. W. et al. Motility abnormalities in irritable bowel syndrome. Digestion 89, 119-123 (2014).

79. Camilleri, M. et al. Prospective study of motor, sensory, psychologic, and autonomic functions in patients with irritable bowel syndrome. Clin. Gastroenterol. Hepatol. 6, 772-781 (2008).

80. Benninga, M. A. et al. Defaecation disorders in children, colonic transit time versus the Barr-score. Eur. J. Pediatr. 154, 277-284 (1995).

81. Rodriguez, L., Sood, M., Di Lorenzo, C. \& Saps, M. An ANMS-NASPGHAN consensus document on anorectal and colonic manometry in children. Neurogastroenterol. Motil. https://doi.org/10.1111 nmo. 12944 (2017)

82. Van Ginkel, R., Voskuijl, W. P., Benninga, M. A., Taminiau, J. A. \& Boeckxstaens, G. E. Alterations in rectal sensitivity and motility in childhood irritable bowel syndrome. Gastroenterology 120, 31-38 (2001).

83. Devanarayana, N. M. \& Rajindrajith, S. Irritable bowe syndrome in children: current knowledge, challenges and opportunities. World J. Gastroenterol. 24, 2211-2235 (2018).

84. Enck, P. \& Mazurak, N. Dysbiosis in functional bowel disorders. Ann. Nutr. Metab. 72, 296-306 (2018)

85. Enck, P. et al. Irritable bowel syndrome. Nat. Rev. Dis. Primers 2, 16014 (2016)

86. Pittayanon, R. et al. Gut microbiota in patients with irritable bowel syndrome-a systematic review. Gastroenterology 157, 97-108 (2019) This systematic review emphasizes inconsistencies in methodology, analyses and findings between studies and the heterogeneity of microbiota in subjects with IBS.

87. Miquel, S. et al. Faecalibacterium prausnitzii and human intestinal health. Curr. Opin. Microbiol. 16, 255-261 (2013) 
88. Chumpitazi, B. P. et al. Randomised clinical trial: gut microbiome biomarkers are associated with clinical response to a low FODMAP diet in children with the irritable bowel syndrome. Aliment. Pharmacol. Ther 42, 418-427 (2015)

89. Rossi, M. et al. Volatile organic compounds in feces associate with response to dietary intervention in patients with irritable bowel syndrome. Clin. Gastroenterol. Hepatol. 16, 385-391.e1 (2018).

90. Dong, T. S. \& Gupta, A. Influence of early life, diet, and the environment on the microbiome. Clin Gastroenterol. Hepatol 17, 231-242 (2019).

91. Carding, S. R., Davis, N. \& Hoyles, L. Review article: the human intestinal virome in health and disease. Aliment. Pharmacol. Ther. 46, 800-815 (2017).

92. Mukhopadhya, I., Segal, J. P., Carding, S. R., Hart, A. L. $\Sigma$ Hold, G. L. The gut virome: the 'missing link' between gut bacteria and host immunity? Ther. Adv. Gastroenterol. 12, 1756284819836620 (2019).

93. Botschuijver, S. et al. Intestinal fungal dysbiosis is associated with visceral hypersensitivity in patients with irritable bowel syndrome and rats. Gastroenterology 153, 1026-1039 (2017).

94. Sokol, H. et al. Fungal microbiota dysbiosis in IBD. Gut 66, 1039-1048 (2017)

95. Carlson, M. J., Moore, C. E., Tsai, C. M., Shulman, R. J. \& Chumpitazi, B. P. Child and parent perceived foodinduced gastrointestinal symptoms and quality of life in children with functional gastrointestinal disorders. J. Acad. Nutr. Diet. 114, 403-413 (2014).

96. Chumpitazi, B. P., Weidler, E. M., Lu, D. Y., Tsai, C. M. ¿ Shulman, R. J. Self-perceived food intolerances are common and associated with clinical severity in childhood irritable bowel syndrome. J. Acad. Nutr. Diet. 116, 1458-1464 (2016)

97. Reed-Knight, B., Squires, M., Chitkara, D. K. \& van Tilburg, M. A. Adolescents with irritable bowel syndrome report increased eating-associated symptoms, changes in dietary composition, and altered eating behaviors: a pilot comparison study to healthy adolescents. Neurogastroenterol. Motil. 28 1915-1920 (2016)

98. Mouchli, M. A. et al. Evaluating the safety and the effects on colonic compliance of neostigmine during motility testing in patients with chronic constipation Neurogastroenterol. Motil. 28, 871-878 (2016).

99. Pawlowska, K., Umlawska, W. \& Iwanczak, B. A link between nutritional and growth states in pediatric patients with functional gastrointestinal disorders. J. Pediatr. 199, 171-177 (2018)

100. Bohn, L., Storsrud, S. \& Simren, M. Nutrient intake in patients with irritable bowel syndrome compared with the general population. Neurogastroenterol. Motil. 25, 23-30.e1 (2013)

101. Murray, K. et al. Differential effects of FODMAPs (fermentable oligo-, di-, mono-saccharides and polyols) on small and large intestinal contents in healthy subjects shown by MRI. Am. J. Gastroenterol. 109 , 110-119 (2014).

102. van Tilburg, M. A. L. In: Neurogastroenterology: Gastrointestinal motility and functional disorders in children (eds Faure, C., Thapar, N., \& Di Lorenzo, C.), 71-80 (Springer, 2017).

103. Kennedy, P. J., Cryan, J. F., Dinan, T. G. \& Clarke, G. Irritable bowel syndrome: a microbiome-gut-brain axis disorder? World J. Gastroenterol. 20, 14105-14125 (2014)

104. Wang, H., Lee, I. S., Braun, C. \& Enck, P. Effect of probiotics on central nervous system functions in animals and humans - a systematic review. J. Neurogastroenterol. Motil. 22, 589-605 (2016).

105. Dinan, T G \& Cryan, J. F The microbiome-gut-brain axis in health and disease. Gastroenterol. Clin. North. Am. 46, 77-89 (2017)

106. Pinto-Sanchez, M. I. et al. Probiotic Bifidobacterium longum NCC3001 reduces depression scores and alters brain activity: a pilot study in patients with irritable bowel syndrome. Gastroenterology 153 448-459.e8 (2017).

107. Ranasinghe, N., Devanarayana, N. M., Benninga, M. A., van Dijk, M. \& Rajindrajith, S. Psychological maladjustment and quality of life in adolescents with constipation. Arch. Dis. Child. 102, 268-273 (2017)

108. Koloski, N. A., Jones, M. \& Talley, N. J. Evidence that independent gut-to-brain and brain-to-gut pathways operate in the irritable bowel syndrome and functional dyspepsia: a 1-year population-based prospective study. Aliment. Pharmacol. Ther. 44, 592-600 (2016)

109. van Tilburg, M. A. L. \& Carter, C. A. Integration of biomedical and psychosocial treatments in pediatrics functional gastrointestinal disorders. Gastroenterol. Clin. North. Am. 47, 863-875 (2018)

110. Hollier, J. M. et al. Multiple psychological factors predict abdominal pain severity in children with irritable bowel syndrome. Neurogastroenterol. Motil. 31, e13509 (2019).

111. Levy, R. L. et al. Cognitive mediators of treatment outcomes in pediatric functional abdominal pain. Clin. J. Pain 30, 1033-1043 (2014). This randomized controlled trial of psychological treatment found no role of anxiety on pain; instead, child pain catastrophizing and parentperceived threat regarding their child's pain predicted reductions in child abdominal pain

112. Bonnert, M. et al. The role of avoidance behavior in the treatment of adolescents with irritable bowe syndrome: a mediation analysis. Behav. Res. Ther. 105, 27-35 (2018)

113. Siawash, M. et al. Abdominal wall pain or irritable bowel syndrome: validation of a pediatric questionnaire. J. Pediatr. Gastroenterol. Nutr. 69 e65-e69 (2019).

114. Lane, M. M., Weidler, E. M., Czyzewski, D. I. \& Shulman, R. J. Pain symptoms and stooling patterns do not drive diagnostic costs for children with functional abdominal pain and irritable bowel syndrome in primary or tertiary care. Pediatrics 123 758-764 (2009).

115. Turco, R. et al. The association of coeliac disease in childhood with functional gastrointestinal disorders: a prospective study in patients fulfilling Rome III criteria. Aliment. Pharmacol. Ther 34, 783-789 (2011).

116. Saps, M. et al. Abdominal pain-associated functional gastrointestinal disorder prevalence in children and adolescents with celiac disease on gluten-free diet: a multinational study. J. Pediatr. 182, 150-154 (2017).

117. Heida, A., Holtman, G. A., Lisman-van Leeuwen, Y., Berger, M. Y. \& van Rheenen, P. F. Avoid endoscopy in children with suspected inflammatory bowel disease who have normal calprotectin levels. J. Pediatr. Gastroenterol. Nutr. 62, 47-49 (2016).

118. Macarthur, C. Helicobacter pylori infection and childhood recurrent abdominal pain: lack of evidence for a cause and effect relationship. Can. J. Gastroenterol. 13, 607-610 (1999).

119. Trivic, I. \& Hojsak, I. Initial diagnosis of functional gastrointestinal disorders in children increases a chance for resolution of symptoms. Pediatr Gastroenterol. Hepatol. Nutr. 21, 264-270 (2018) An important observational study, which implies that a prompt diagnosis (at the first visit) based on symptoms and clinical history leads to a sufficient outcome in children presenting with a functional gastrointestinal disorder. It implies that testing is often unnecessary to establish a firm diagnosis.

120. Bonilla, S., Deli, W. \& Saps, M. The prognostic value of obtaining a negative endoscopy in children with functional gastrointestinal disorders. Clin. Pediatr. $\mathbf{5 0}$ 396-401 (2011)

121. Freedman, S. B. et al. Delayed diagnoses in children with constipation: multicenter retrospective cohort study. J. Pediatr. 186, 87-94.e16 (2017).

122. van Tilburg, M. A. et al. Parents' worries about recurrent abdominal pain in children. Gastroenterol. Nurs. 29, 50-55 (2006).

123. Santucci, N. R., Saps, M. \& van Tilburg, M. A. New advances in the treatment of paediatric functiona abdominal pain disorders. Lancet Gastroenterol. Hepatol. 5, 316-328 (2020)

124. Alfaro Cruz, L., Minard, C., Guffey, D., Chumpitazi, B. P. $\&$ Shulman, R. J. Does a minority of children with functional gastrointestinal disorders receive formal diet advice? JPEN J Parenter. Enter Nutr. https://doi. org/10.1002/jpen.1771 (2020)

125. Duncanson, K. R., Talley, N. J., Walker, M. M. \& Burrows, T. L. Food and functional dyspepsia: a systematic review. J. Hum. Nutr. Diet. 31, 390-407 (2018).

126. Duboc, H., Latrache, S., Nebunu, N. \& Coffin, B. The role of diet in functional dyspepsia management. Front. Psychiatry 11, 23 (2020)

127. Barrett, J. S. \& Gibson, P. R. Clinical ramifications of malabsorption of fructose and other short-chain carbohydrates. Pract. Gastroenterol. 31, 51-65 (2007).

128. Halmos, E. P., Power, V. A., Shepherd, S. J., Gibson, P. R. $\varangle$ Muir, J. G. A diet low in FODMAPs reduces symptom of irritable bowel syndrome. Gastroenterology 146 67-75.e5 (2014).

129. Chumpitazi, B. P. et al. Fructans exacerbate symptoms in a subset of children with irritable bowel syndrome Clin. Gastroenterol. Hepatol. 16, 219-225.e1 (2018).
130. Shepherd, S. J., Parker, F. C., Muir, J. G. \& Gibson, P. R. Dietary triggers of abdominal symptoms in patients with irritable bowel syndrome: randomized placebocontrolled evidence. Clin. Gastroenterol. Hepatol. 6 765-771 (2008)

131. Major, G. et al. Colon hypersensitivity to distension, rather than excessive gas production, produces carbohydrate-related symptoms in individuals with irritable bowel syndrome. Gastroenterology 152 , 124-133.e2 (2017).

This study underscores how certain foods may induce symptoms in patients with IBS by causing visceral hypersensitivity that can be activated by distention and, based on other studies, by food-induced inflammation.

132. Yang, J. et al. Lactose intolerance in irritable bowel syndrome patients with diarrhoea: the roles of anxiety, activation of the innate mucosal immune system and visceral sensitivity. Aliment. Pharmacol. Ther. 39, 302-311 (2014).

133. Zhou, S. Y. et al. FODMAP diet modulates visceral nociception by lipopolysaccharide-mediated intestinal inflammation and barrier dysfunction. J. Clin. Invest. 128, 267-280 (2018)

134. Halmos, E. P. \& Gibson, P. R. Controversies and reality of the FODMAP diet for patients with irritable bowel syndrome. J. Gastroenterol. Hepatol. 34, 1134-1142 (2019).

135. Wilder-Smith, C. H., Olesen, S. S., Materna, A. $\&$ Drewes, A. M. Predictors of response to a low-FODMAP diet in patients with functional gastrointestinal disorders and lactose or fructose intolerance. Aliment. Pharmacol. Ther. 45 1094-1106 (2017).

136. Bellini, M. et al. Low FODMAP diet: evidence, doubts, and hopes. Nutrients 12, 148 (2020)

137. Lebenthal, E., Rossi, T. M., Nord, S. K. \& Branski, D. Recurrent abdominal pain and lactose absorption in children. Pediatrics 67, 828-832 (1981).

138. Dearlove, J., Dearlove, B., Pearl, K. \& Primavesi, R. Dietary lactose and the child with abdominal pain. Br. Med. J. 286, 1936-1936 (1983).

139. Garcia-Etxebarria, K. et al. Increased prevalence of rare sucrase-isomaltase pathogenic variants in irritable bowel syndrome patients. Clin. Gastroenterol. Hepatol. 16, 1673-1676 (2018)

140. Gericke, B., Amiri, M., Scott, C. R. \& Naim, H. Y. Molecular pathogenicity of novel sucrase-isomaltase mutations found in congenital sucrase-isomaltase deficiency patients. Biochim. Biophys. Acta Mol. Basis Dis. 1863, 817-826 (2017)

141. Zheng, T. et al. Reduced efficacy of low FODMAPs diet in patients with IBS-D carrying sucrase-isomaltase (SI) hypomorphic variants. Gut 69, 397-398 (2019).

142. Fritscher-Ravens, A. et al. Confocal endomicroscopy shows food-associated changes in the intestinal mucosa of patients with irritable bowel syndrome. Gastroenterology 147, 1012-1020.e4 (2014).

143. Fritscher-Ravens, A. et al. Many patients with irritable bowel syndrome have atypical food allergies not associated with immunoglobulin E. Gastroenterology 157, 109-118.e5 (2019).

144. Boyce, J. A. et al. Guidelines for the diagnosis and management of food allergy in the United States: summary of the NIAID-sponsored expert panel report. Nutr. Res. 31, 61-75 (2011)

145. Azcarate-Peril, M. A. et al. Impact of short-chain galactooligosaccharides on the gut microbiome of lactose-intolerant individuals. Proc. Natl Acad. Sci. USA 114, E367-E375 (2017).

146. Huaman, J. W. et al. Effects of prebiotics vs a diet low in FODMAPs in patients with functional gut disorders. Gastroenterology 155, 1004-1007 (2018).

147. Medow, M. S. et al. Beta-galactosidase tablets in the treatment of lactose intolerance in pediatrics. Am. J. Dis. Child. 144, 1261-1264 (1990).

148. Treem, W. R. Clinical aspects and treatment of congenital sucrase-isomaltase deficiency. J. Pediatr. Gastroenterol. Nutr. 55 (Suppl. 2), S7-S13 (2012).

149. Komericki, P. et al. Oral xylose isomerase decrease breath hydrogen excretion and improves gastrointestinal symptoms in fructose malabsorption a double-blind, placebo-controlled study. Aliment. Pharmacol. Ther. 36, 980-987 (2012).

150. Tuck, C. J., Taylor, K. M., Gibson, P. R., Barrett, J. S $\&$ Muir, J. G. Increasing symptoms in irritable bowel symptoms with ingestion of galacto-oligosaccharides are mitigated by alpha-galactosidase treatment. Am. J. Gastroenterol. 113, 124-134 (2018)

151. Huang, R. C., Palmer, L. J. \& Forbes, D. A. Prevalence and pattern of childhood abdominal pain in an 
Australian general practice. J. Paediatr. Child. Health 36, 349-353 (2000)

152. Paulo, A. Z., Amancio, O. M., de Morais, M. B. \& Tabacow, K. M. Low-dietary fiber intake as a risk factor for recurrent abdominal pain in children. Eur. J. Clin. Nutr. 60, 823-827 (2006).

153. Moayyedi, P. et al. The effect of fiber supplementation on irritable bowel syndrome: a systematic review and meta-analysis. Am. J. Gastroenterol. 109 1367-1374 (2014).

154. Nagarajan, N. et al. The role of fiber supplementation in the treatment of irritable bowel syndrome: a systematic review and meta-analysis. Eur. $J$. Gastroenterol. Hepatol. 27, 1002-1010 (2015).

155. Eswaran, S., Muir, J. \& Chey, W. D. Fiber and functional gastrointestinal disorders. Am. J. Gastroenterol. 108, 718-727 (2013).

156. Shulman, R. J. et al. Psyllium fiber reduces abdominal pain in children with irritable bowel syndrome in a randomized, double-blind trial. Clin. Gastroenterol. Hepatol. 15, 1915-1920.e4 (2017).

157. Ford, A. C., Harris, L. A., Lacy, B. E., Quigley, E. M. M. \& Moayyedi, P. Systematic review with meta-analysis: the efficacy of prebiotics, probiotics, synbiotics and antibiotics in irritable bowel syndrome. Aliment. Pharmacol. Ther. 48, 1044-1060 (2018).

158. Wegh, C. A. M., Benninga, M. A. \& Tabbers, M. M Effectiveness of probiotics in children with functional abdominal pain disorders and functional constipation: a systematic review. J. Clin. Gastroenterol. 52, (Suppl. 1), S10-S26 (2018).

159. Cheung, W. Y. et al. The economic impact of the transition from branded to generic oncology drugs. Curr. Oncol. 26, 89-93 (2019)

160. Romano, C. et al. Lactobacillus reuteri in children with functional abdominal pain (FAP). J. Paediatr. Child. Health 50, E68-E71 (2014).

161. Weizman, Z., Abu-Abed, J. \& Binsztok, M. Lactobacillus reuteri DSM 17938 for the management of functional abdominal pain in childhood: a randomized, double-blind, placebocontrolled trial. J. Pediatr. https://doi.org/10.1016/ j.jpeds.2016.04.003 (2016).

162. Jadresin, O. et al. Lactobacillus reuteri DSM 17938 in the treatment of functional abdominal pain in children - RCT study. J. Pediatr. Gastroenterol. Nutr. 64, 925-929 (2017).

163. Maragkoudaki, M. et al. Lactobacillus reuteri DSM 17938 and a placebo both significantly reduced symptoms in children with functional abdominal pain. Acta Paediatr. 106, 1857-1862 (2017).

164. Eftekhari, K., Vahedi, Z., Kamali Aghdam, M. \& Diaz, D. N. A randomized double-blind placebocontrolled trial of lactobacillus reuteri for chronic functional abdominal pain in children. Iran. J. Pediatr. 25, e2616 (2015)

165. Francavilla, R. et al. A randomized controlled trial of Lactobacillus GG in children with functional abdominal pain. Pediatrics 126, e1445-e1452 (2010)

166. Gawronska, A., Dziechciarz, P., Horvath, A. \& Szajewska, H. A randomized double-blind placebocontrolled trial of Lactobacillus GG for abdominal pain disorders in children. Aliment. Pharmacol. Ther. 25, 177-184 (2007)

167. Guandalini, S. et al. VSL\#3 improves symptoms in children with irritable bowel syndrome: a multicenter, randomized, placebo-controlled, double-blind, crossover study. J. Pediatr. Gastroenterol. Nutr. 51, 24-30 (2010).

168. Lackner, J. M. et al. Durability and decay of treatment benefit of cognitive behavioral therapy for irritable bowel syndrome: 12-month follow-up. Am. J. Gastroenterol. 114, 330-338 (2019).

169. Lackner, J. M. et al. Improvement in gastrointestinal symptoms after cognitive behavior therapy for refractory irritable bowel syndrome. Gastroenterology 155, 47-57 (2018)

170. Rutten, J. M. T. M., Korterink, J. J., Venmans, L. M. A. J., Benninga, M. A. \& Tabbers, M. M. Nonpharmacologic treatment of functional abdominal pain disorders: a systematic review. Pediatrics 135, 522-535 (2015).

A large randomized controlled trial showing the (long-term) efficacy of hypnotherapy by a therapist and by home-based hypnotherapy self-exercises. The home-based hypnotherapy is cheap and may be available in many languages in the near future.

171. Abbott, R. A. et al. Recurrent abdominal pain in children: summary evidence from 3 systematic reviews of treatment effectiveness. J. Pediatr. Gastroenterol. Nutr. 67, 23-33 (2018)
172. Levy, R. L. et al. Cognitive-behavioral therapy for children with functional abdominal pain and their parents decreases pain and other symptoms. Am. J. Gastroenterol. 105, 946-956 (2010)

173. Levy, R. L. et al. Twelve-month follow-up of cognitive behavioral therapy for children with functional abdominal pain. JAMA Pediatr. 167, 178-184 (2013).

174. Lalouni, M. et al. Clinical and cost effectiveness of online cognitive behavioral therapy in children with functional abdominal pain disorders. Clin. Gastroenterol. Hepatol. 17, 2236-2244.e11 (2019).

175. Levy, R. L. et al. Brief telephone-delivered cognitive behavioral therapy targeted to parents of children with functional abdominal pain: a randomized controlled trial Pain 158, 618-628 (2017).

176. Bonnert, M. et al. Internet-delivered cognitive behavior therapy for adolescents with irritable bowel syndrome: a randomized controlled trial. Am. J. Gastroenterol. 112, 152-162 (2017).

177. Sampaio, F. et al. Cost-effectiveness of internetdelivered cognitive-behavioural therapy for adolescents with irritable bowel syndrome. BMJ Open 9, e023881 (2019)

178. Reed-Knight, B., Claar, R. L., Schurman, J. V. \& van Tilburg, M. A. Implementing psychological therapies for functional $\mathrm{GI}$ disorders in children and adults. Expert. Rev. Gastroenterol. Hepatol. 10 981-984 (2016)

179. Prior, A., Colgan, S. M. \& Whorwell, P. J. Changes in rectal sensitivity after hypnotherapy in patients with irritable bowel syndrome. Gut 31, 896-898 (1990).

180. Whorwell, P. J., Houghton, L. A., Taylor, E. E. \& Maxton, D. G. Physiological effects of emotion: assessment via hypnosis. Lancet 340, 69-72 (1992)

181. Palsson, O. S., Turner, M. J., Johnson, D. A., Burnett, C. K. $\&$ Whitehead, W. E. Hypnosis treatment for severe irritable bowel syndrome: investigation of mechanism and effects on symptoms. Dig. Dis. Sci. 47 2605-2614 (2002).

182. Vlieger, A. M. et al. No change in rectal sensitivity after gut-directed hypnotherapy in children with functional abdominal pain or irritable bowel syndrome. Am. J. Gastroenterol. 105, 213-218 (2010).

183. Vlieger, A. M., Rutten, J. M., Govers, A. M., Frankenhuis, C. \& Benninga, M. A. Long-term followup of gut-directed hypnotherapy vs. standard care in children with functional abdominal pain or irritable bowel syndrome. Am. J. Gastroenterol. 107, 627-631 (2012).

184. Rutten, J. et al. Home-based hypnotherapy selfexercises vs individual hypnotherapy with a therapist for treatment of pediatric irritable bowel syndrome, functional abdominal pain, or functional abdominal pain syndrome: a randomized clinical trial. JAMA Pediatr. 171, 470-477 (2017).

185. Korterink, J. J., Ockeloen, L. E., Hilbink, M., Benninga, M. A. \& Deckers-Kocken, J. M. Yoga therapy for abdominal pain-related functional gastrointestinal disorders in children: a randomized controlled trial. J. Pediatr. Gastroenterol. Nutr. 63, 481-487 (2016)

186. Bittar, R. G. et al. Deep brain stimulation for pain relief: a meta-analysis. J. Clin. Neurosci. 12, 515-519 (2005).

187. Babygirija, R., Sood, M., Kannampalli, P. Sengupta, J. N. \& Miranda, A. Percutaneous electrical nerve field stimulation modulates central pain pathways and attenuates post-inflammatory visceral and somatic hyperalgesia in rats. Neuroscience 356 11-21 (2017).

188. Kovacic, K. et al. Neurostimulation for abdominal pain-related functional gastrointestinal disorders in adolescents: a randomised, double-blind, shamcontrolled trial. Lancet Gastroenterol. Hepatol. 2 727-737 (2017)

189. Day, A. S. Use of complementary and alternative therapies and probiotic agents by children attending gastroenterology outpatient clinics. J. Paediatr. Child. Health 38, 343-346 (2002).

190. Vlieger, A. M., Blink, M., Tromp, E. \& Benninga, M. A Use of complementary and alternative medicine by pediatric patients with functional and organic gastrointestinal diseases: results from a multicenter survey. Pediatrics 122, e446-e451 (2008).

191. Korterink, J. J., Rutten, J. M., Venmans, L. Benninga, M. A. \& Tabbers, M. M. Pharmacologic treatment in pediatric functional abdominal pain disorders: a systematic review. J. Pediatr. 166 424-431.e6 (2015).
192. Elsenbruch, S. \& Enck, P. Placebo effects and their determinants in gastrointestinal disorders. Nat. Rev. Gastroenterol. Hepatol. 12, 472-485 (2015).

193. Weimer, K. et al. Placebo effects in children: a review. Pediatr. Res. 74, 96-102 (2013).

194. Czerniak, E., Oberlander, T. F., Weimer, K., Kossowsky, J. \& Enck, P. "Placebo by proxy" and "Nocebo by proxy" in children: a review of parents' role in treatment outcomes. Front. Psychiatry 11, 169 (2020).

195. Rome Foundation. Gl genius interactive clinical decision toolkit. Rome Foundation https://romeonline. $\mathrm{org} /$ product/rome-iv-interactive-clinical-decisionoolkit-logicnets (2020)

196. Kline, R. M., Kline, J. J., Di Palma, J. \& Barbero, G. J. Enteric-coated, $\mathrm{pH}$-dependent peppermint oil capsules for the treatment of irritable bowel syndrome in children. J. Pediatr. 138, 125-128 (2001).

197. Saps, M., Miranda, A. in Gastrointestinal Pharmacology Vol. 239 (ed. Greenwood-Van Meerveld, B.) 147-176 (Springer, 2017).

198. Karabulut, G. S. et al. The incidence of irritable bowel syndrome in children using the Rome III criteria and the effect of trimebutine treatment. J. Neurogastroenterol. Motil. 19, 90-93 (2013).

199. Pourmoghaddas, Z., Saneian, H., Roohafza, H. \& Gholamrezaei, A. Mebeverine for pediatric functional abdominal pain: a randomized, placebo-controlled trial. Biomed. Res. Int. 2014, 191026 (2014).

200. Narang, M., Shah, D. \& Akhtar, H. Efficacy and safety of drotaverine hydrochloride in children with recurrent abdominal pain: a randomized placebo controlled trial. Indian Pediatr. 52, 847-851 (2015).

201. Scarpellini, E. et al. Rifaximin treatment for small intestinal bacterial overgrowth in children with irritable bowel syndrome. Eur. Rev. Med. Pharmacol. Sci. 17, 1314-1320 (2013).

202. Collins, B. S. \& Lin, H. C. Double-blind, placebocontrolled antibiotic treatment study of small intestinal bacterial overgrowth in children with chronic abdominal pain. J. Pediatr. Gastroenterol. Nutr. 52 , 382-386 (2011)

203. Bahar, R. J., Collins, B. S. Steinmetz, B. \& Ament, M. E. Double-blind placebo-controlled trial of amitriptyline for the treatment of irritable bowel syndrome in adolescents. J. Pediatr. 152, 685-689 (2008).

204. Saps, M. et al. Multicenter, randomized, placebocontrolled trial of amitriptyline in children with functional gastrointestinal disorders. Gastroenterology 137, 1261-1269 (2009).

205. Campo, J V et al. Citalopram treatment of pediatric recurrent abdominal pain and comorbid internalizing disorders: an exploratory study. J. Am. Acad. Child. Adolesc. Psychiatry 43, 1234-1242 (2004).

206. Roohafza, H., Pourmoghaddas, Z., Saneian, H. \& Gholamrezaei, A. Citalopram for pediatric functiona abdominal pain: a randomized, placebo-controlled trial. Neurogastroenterol. Motil. 26, 1642-1650 (2014).

207. Federal Drug Administration (FDA). Suicidality in children and adolescents being treated with antidepressant medications. FDA https://www.fda.gov/ drugs/postmarket-drug-safety-information-patientsand-providers/suicidality-children-and-adolescentsbeing-treated-antidepressant-medications (2018)

208. Lee, K. J., Kim, J. H. \& Cho, S. W. Gabapentin reduces rectal mechanosensitivity and increases rectal compliance in patients with diarrhoea-predominant irritable bowel syndrome. Aliment. Pharmacol. Ther 22, 981-988 (2005).

209. Houghton, L. A et al. Effect of a second-generation alpha2delta ligand (pregabalin) on visceral sensation in hypersensitive patients with irritable bowel syndrome. Gut 56, 1218-1225 (2007).

210. Mugie, S. M. et al. Prucalopride is no more effective than placebo for children with functional constipation. Gastroenterology 147, 1285-1295.e1 (2014).

211. Benninga, M. et al. Efficacy and safety of lubiprostone in children with functional constipation: a multicenter randomized, placebo-controlled, double-blind pivotal study. Gastroenterology 154, S559-S560 (2018).

212. Lisman-van Leeuwen, Y., Spee, L. A. A., Benninga, M. A Bierma-Zeinstra, S. M. A. \& Berger, M. Y. Prognosis of abdominal pain in children in primary care-a prospective cohort study. Ann. Fam. Med. 11, 238-244 (2013)

213. Saps, M. et al. Recommendations for pharmacological clinical trials in children with irritable bowel syndrome: the Rome foundation pediatric subcommittee on clinical trials. Neurogastroenterol. Motil. 28, 1619-1631 (2016). 
214. Federal Drug Administration (FDA). Gastroenterology regulatory endpoints and the advancement of therapeutics for alcoholic hepatitis and alcohol associated liver disease and pediatric irritable bowe syndrome and pediatric functional constipation workshop. FDA https://www.fda.gov/drugs/newsevents-human-drugs/gastroenterology-regulatoryendpoints-and-advancement-therapeutics-alcoholichepatitis-and-alcohol (2020)

215. Federal Drug Administration (FDA). Patient-Reported Outcome Measures: Use in Medical Product Development to Support Labeling Claims. (US Department of Health and Human Services, 2009).

216. Riley, A. W. Evidence that school-age children can self-report on their health. Ambul. Pediatr. 4 , 371-376 (2004).

217. Varni, J. W. et al. Gastrointestinal symptoms predictors of health-related quality of life in pediatric patients with functional gastrointestinal disorders. Qual. Life Res. 26, 1015-1025 (2017).

218. Irwin, D. E. et al. Sampling plan and patient characteristics of the PROMIS pediatrics large-scale survey. Qual. Life Res. 19, 585-594 (2010).

219. Spiegel, B. M. et al. Development of the NIH patient-reported outcomes measurement information system (PROMIS) gastrointestinal symptom scales. Am. J. Gastroenterol 109, 1804-1814 (2014).

220. Varni, J. W. The PedsQL (TM) measurement model for the pediatric quality of life inventory (TM). Peds $Q L$ http://www. pedsql.org (2020).

221. Varni, J. W., Seid, M., Knight, T. S., Uzark, K. \& Szer, I. S. The PedsOL 4.0 generic core scales: sensitivity, responsiveness, and impact on clinical decision-making. J. Behav. Med. 25, 175-193 (2002).

222. Varni, J. W., Seid, M. \& Kurtin, P. S. PedsQL 4.0: reliability and validity of the pediatric quality of life inventory version 4.0 generic core scales in healthy and patient populations. Med. Care 39, 800-812 (2001).

223. Varni, J. W., Seid, M. \& Rode, C. A. The PedsQL measurement model for the pediatric quality of life inventory. Med. Care 37, 126-139 (1999).

224. Varni, J. W., Kay, M. T., Limbers, C. A., Franciosi, J. P. $\&$ Pohl, J. F. PedsOL gastrointestinal symptoms module item development: qualitative methods J. Pediatr. Gastroenterol. Nutr. 54, 664-671 (2012).

225. Varni, J. W. et al. PedsQL gastrointestinal symptoms module: feasibility, reliability, and validity. J. Pediatr. Gastroenterol. Nutr. 59, 347-355 (2014).

226. Varni, J. W. et al. Health-related quality of life in pediatric patients with functional and organic gastrointestinal diseases. J. Pediatr. 166, 85-90 (2015)

227. Varni, J. W. et al. PedsQL gastrointestinal symptoms scales and gastrointestinal worry scales in pediatric patients with functional and organic gastrointestinal diseases in comparison to healthy controls. Qual. Life Res. 24, 363-378 (2015)

228. van Tilburg, M. A. \& Murphy, T. B. Quality of life paradox in gastrointestinal disorders. J. Pediatr. 166, 11-14 (2015).

229. Walker, L. S. ¿ Greene, J. W. The functional disability inventory: measuring a neglected dimension of child health status. J. Pediatr. Psychol. 16, 39-58 (1991).

230. Claar, R. L. \& Walker, L. S. Functional assessment of pediatric pain patients: psychometric properties of the functional disability inventory. Pain 121, 77-84 (2006).

231. Russell, A. C., Stone, A. L. \& Walker, L. S. Nausea in children with functional abdominal pain predicts poor health outcomes in young adulthood. Clin. Gastroenterol. Hepatol. 15, 706-711 (2017).

232. Campo, J. V. et al. Adult outcomes of pediatric recurrent abdominal pain: do they just grow out of it? Pediatrics 108, E1 (2001).
233. Shelby, G. D. et al. Functional abdominal pain in childhood and long-term vulnerability to anxiety disorders. Pediatrics 132, 475-482 (2013).

234. Hoge, E. A., Pollack, M. H., Kaufman, R. E., Zak, P. J. $\&$ Simon, N. M. Oxytocin levels in social anxiety disorder. CNS Neurosci. Ther. 14, 165-170 (2008).

235. Gieteling, M. J., Bierma-Zeinstra, S. M., Passchier, J. $\Sigma$ Berger, M. Y. Prognosis of chronic or recurrent abdominal pain in children. J. Pediatr. Gastroenterol. Nutr. 47, 316-326 (2008).

236. Horst, S. et al. Predicting persistence of functional abdominal pain from childhood into young adulthood. Clin. Gastroenterol. Hepatol. 12, 2026-2032 (2014).

237. Lindley, K. J., Glaser, D. \& Milla, P. J. Consumerism in healthcare can be detrimental to child health: lessons from children with functional abdominal pain Arch. Dis. Child. 90, 335-337 (2005).

238. Pace, F. et al. Family history of irritable bowel syndrome is the major determinant of persistent abdominal complaints in young adults with a history of pediatric recurrent abdominal pain. World $\mathrm{J}$. Gastroenterol. 12, 3874-3877 (2006).

239. Dengler-Crish, C. M., Horst, S. N. \& Walker, L. S. Somatic complaints in childhood functional abdominal pain are associated with functional gastrointestinal disorders in adolescence and adulthood. J. Pediatr. Gastroenterol. Nutr. 52, 162-165 (2011).

240. Feinle-Bisset, C. \& Azpiroz, F. Dietary and lifestyle factors in functional dyspepsia. Nat. Rev. Gastroenterol. Hepatol. 10, 150-157 (2013).

241. Krasaelap, A. \& Madani, S. Cyproheptadine: a potentially effective treatment for functional gastrointestinal disorders in children. Pediatr. Ann. 46, e120-e125 (2017)

242. Rodriguez, L., Diaz, J. \& Nurko, S. Safety and efficacy of cyproheptadine for treating dyspeptic symptoms in children. J. Pediatr. 163, 261-267 (2013).

243. Li, J. et al. A combination of peppermint oil and caraway oil for the treatment of functional dyspepsia: a systematic review and meta-analysis. Evid. Based Complement. Altern. Med. 2019, 7654947 (2019)

244. Kim, Y. S., Kim, J.-W., Ha, N.-Y., Kim, J. \& Ryu, H. S. Herbal therapies in functional gastrointestinal disorders: a narrative review and clinical implication. Front. Psychiatry 11, 601 (2020).

245. Browne, P. D., Nagelkerke, S. C. J., van EttenJamaludin, F. S., Benninga, M. A. \& Tabbers, M. M. Pharmacological treatments for functional nausea and functional dyspepsia in children: a systematic review. Expert. Rev. Clin. Pharmacol. https://doi.org/10.1080/ 17512433.2018.1540298 (2018)

246. Masuy, I., Van Oudenhove, L. \& Tack, J. Review article treatment options for functional dyspepsia. Aliment Pharmacol. Ther. 49, 1134-1172 (2019).

247. Li, B. U. K. Managing cyclic vomiting syndrome in children: beyond the guidelines. Eur. J. Pediatr. 177 1435-1442 (2018).

248. Beinvogl, B. et al. Multidisciplinary treatment reduces pain and increases function in children with functional gastrointestinal disorders. Clin. Gastroenterol. Hepatol. 17, 994-996 (2019).

249. Dehghani, S. M., Imanieh, M. H., Oboodi, R. $\&$ Haghighat, M. The comparative study of the effectiveness of cimetidine, ranitidine, famotidine, and omeprazole in treatment of children with dyspepsia. ISRN Pediatr. 2011, 219287 (2011).

250. KIDSCREEN. Health-related quality of life questionnaire for children and adolescents aged from 8 to 18 years. KIDSCREEN https://www.kidscreen.org/english/ questionnaires (2020)

251. KINDL. KINDL: revised questionnaire for CHILDREN and adolescents to record the health-related quality of life. KINDL https://www.kindl.org/ (2020)

252. GCO. Generic children's quality of life measure CORC https://www.corc.uk.net/outcome-experience- measures/generic-childrens-quality-of-life-measure/ (2020).

253. HUInc. Health Utilities Inc: health-related qualityof-life. HUInc http://healthutilities.com (2020).

254. HACHO. Infant toddler quality of life questionnaire (ITOOL). Inspiring Impact https://www. inspiringimpact. org/resource-library/infant-toddler-quality-of-lifequestionnaire-itqol/ (2020).

255. Brugman, E. et al. TNO-AZL preschool children quality of life questionnaire (TAPOOL). ePROVIDE https:// eprovide.mapi-trust.org/instruments/tno-azl-preschoolchildren-quality-of-life-questionnaire/ (2020).

256. Fekkes, M. et al. TNO AZL children's quality of life (TACQOL). ePROVIDE https://eprovide.mapi-trust.org/ instruments/tno-azl-children-s-quality-of-life/ (2020).

257. PROMIS. List of pediatric measures. HealthMeasures https://www.healthmeasures.net/exploremeasurement-systems/promis/intro-to-promis/ list-of-pediatric-measures/ (2020).

\section{Acknowledgements}

R.J.S. acknowledges the funding R01 NR013497 and R01 NR016786 from the National Institutes of Health the USDA/ ARS under Cooperative Agreement No. 58-3092-0-001, and P30 DK56338, which funds the Texas Medical Center Digestive Disease Center. The content is solely the responsibility of the authors and does not necessarily represent the official views of the National Institutes of Health. This work is a publication of the USDA/ARS Children's Nutrition Research Center, Department of Paediatrics, Baylor College of Medicine and Texas Children's Hospital. The contents do not necessarily reflect the views or policies of the USDA nor does the mention of trade names, commercial products or organizations imply endorsement by the US Government.

\section{Author contributions}

Introduction (P.E. and N.T.); Epidemiology (P.E., I.M. and N.T.); Mechanisms/pathophysiology (S.N., H.S., M.A.L.v.T. and N.T.); Diagnosis, screening and prevention (C.D.L. and M.A.L.v.T.); Management (M.A.B., M.S. and R.J.S.); Quality of life (M.D.C.); Outlook (N.T.); Overview of Primer (P.E. and N.T.).

\section{Competing interests}

N.T. has received grants, membership on advisory boards, speaker fees or travel support from Danone, Nutricia and Takeda. M.A.B. has received grants, membership on advisory boards, speaker fees or travel support from Abbott, Allergan, Coloplast, Danone, Friesland Campina, HIPP, Johnson \& Johnson, Norgine, Shire, Sucampo and United Pharmaceuticals. C.D.L. has received grants, membership on advisory boards, speaker fees or travel support from Allergan, Mahana, Mallinckrodt, OOL, Sucampo and Takeda. M.S. has received grants, membership on advisory boards, speaker fees or travel support from Abbott, Allergan, Forest, $\mathrm{QOL}$ and Sucampo. H.S. has received grants, membership on advisory boards, speaker fees or travel support from Arla, Biocodex, Biogaia, Ch.Hansen, Danone, Merck, Nestlé and Nutricia. M.A.L.v.T. has received consultancy fees from Mahana. P.E. has received grants, membership on advisory boards, speaker fees or travel support from Alimentary Health, Aptinyx, Arena, Biocodex, CEMET, Danone, Genetic Analysis, Indigo, Merz, MDC, Sanofi, SymbioPharm and 4DPharma. All other authors declare no competing interests.

\section{Peer review information}

Nature Reviews Disease Primers thanks Christopher Eccleston, Anne Lynch-Jordan, Emeran Mayer and the other, anonymous, reviewer(s) for their contribution to the peer review of this work.

\section{Publisher's note}

Springer Nature remains neutral with regard to jurisdictional claims in published maps and institutional affiliations.

C) Springer Nature Limited 2020 